\title{
Moment-to-moment fluctuations in fMRI amplitude and interregion coupling are predictive of inhibitory performance
}

\author{
Srikanth Padmala and Luiz Pessoa \\ Indiana University, Bloomington, Indiana
}

\begin{abstract}
We investigated how moment-to-moment fluctuations in fMRI amplitude and interregional coupling are linked to behavioral performance during a stop signal task. To quantify the relationship between single-trial amplitude and behavior on a trial-by-trial basis, we modeled the probability of successful inhibition as a function of response amplitude via logistic regression analysis. At the group level, significant logistic slopes were observed in, among other regions, the inferior frontal gyrus (IFG), caudate, and putamen, all bilaterally. Furthermore, we investigated how trial-by-trial fluctuations in responses in attentional regions covaried with fluctuations in inhibition-related regions. The coupling between several frontoparietal attentional regions and the right IFG increased during successful versus unsuccessful performance, suggesting that efficacious network interactions are important in determining behavioral outcome during the stop signal task. In particular, the link between responses in the right IFG and behavior were moderated by moment-to-moment fluctuations in evoked responses in the left intraparietal sulcus. A supplemental figure for this article may be downloaded from http:// cabn.psychonomic-journals.org/content/supplemental.
\end{abstract}

Response inhibition, the ability to suppress actions that are no longer behaviorally relevant or contextually appropriate, is a key function of the human executive control system. This function has been investigated behaviorally, with monkey physiology, and with human ERPs and fMRI by using go/no-go (Casey et al., 1997; Eimer, 1993; Kalaska \& Crammond, 1995) and stop signal (Aron et al., 2007; Boucher, Palmeri, Logan, \& Schall, 2007; Li, Huang, Constable, \& Sinha, 2006; Logan, 1994; Logan \& Cowan, 1984) tasks. Response inhibition is believed to involve control regions in the prefrontal cortex (PFC), and both lesion and fMRI studies have suggested that the inferior frontal cortex (IFC), especially on the right hemisphere, is centrally involved in this function (Aron, Fletcher, Bullmore, Sahakian, \& Robbins, 2003; Rubia, Smith, Brammer, \& Taylor, 2003), a notion that is supported by transcranial magnetic stimulation (TMS) studies (Chambers et al., 2007; Chambers et al., 2006). Other studies in the literature have provided evidence for the involvement of additional brain structures in response inhibition, including the presupplementary motor area, superior/medial PFC, and precentral gyrus (Chen, Muggleton, Tzeng, Hung, \& Juan, 2009; Floden \& Stuss, 2006; Li et al., 2006; Nachev, Wydell, O’Neill, Husain, \& Kennard, 2007; Picton et al., 2007). In addition to cortical structures, several subcortical areas have been linked to response inhibition, including the caudate, putamen (Eagle \& Robbins, 2003; Li, Yan, Sinha, \& Lee, 2008; Vink et al.,
2005), and subthalamic nucleus (Aron \& Poldrack, 2006). The latter, in particular, has been suggested to be part of a hyperdirect pathway that includes the IFC and is critical for motor inhibition. Taken together, response inhibition appears to engage a broad constellation of cortical and subcortical sites that are recruited in order to cancel a prepotent response when inhibition is called for (Chambers, Garavan, \& Bellgrove, 2009).

Recent studies have also made the case that network interactions subserve behavioral performance during response inhibition, revealing that multiple inhibitionrelated brain regions simultaneously contribute to this type of behavior (Duann, Ide, Luo, \& Li, 2009). More generally, successful performance during response inhibition is behaviorally challenging and depends on several processes, including perceptual processing and attention, in addition to inhibitory mechanisms per se. Consistent with this notion, a recent MEG study revealed that fluctuations of sensory processing linked to both go and stop stimuli have an impact on inhibitory performance during a stop signal task (Boehler et al., 2009). In a related fashion, several studies have reported the involvement of parietal regions during response inhibition (Garavan, Ross, \& Stein, 1999; Hester, Madeley, Murphy, \& Mattingley, 2009; Liddle, Kiehl, \& Smith, 2001), although the exact nature of their involvement remains to be determined. One possibility is that their role is attentional and, in particular, that they reflect trial-by-trial fluctuations 
in the allocation of resources (see Leber, Turk-Browne, $\&$ Chun, 2008) that are needed for successful behavioral performance during demanding tasks. Overall, interactions between diverse brain regions involved in multiple aspects of task performance may be present during successful response inhibition.

The goals of the present investigation were twofold. First, our aim was to characterize how evoked fMRI responses are linked to behavioral outcome during a response inhibition task on a moment-to-moment basis. Traditionally, both in human fMRI studies and in studies of monkey physiology, two conditions are compared by testing for differences in the associated mean responses. A complementary approach is to investigate how trial-bytrial fluctuations in evoked responses are linked to behavioral performance. Although there is a relation between the information conveyed by these two types of analyses, the trial-by-trial analysis offers a potentially valuable way to quantify the predictive relationship between response magnitude and observed behavior (Figure 1A). For instance, logistic regression analysis can be used to model the probability of a dichotomous behavioral variable (e.g., detected vs. undetected stimulus, correct vs. incorrect performance) as a function of single-trial responses. Trial-based approaches have been used fruitfully in studies investigating perceptual decisions (Padmala \& Pessoa, 2008; Ress, Backus, \& Heeger, 2000), in addition to more cognitive tasks (Leber et al., 2008; Pessoa, Gutierrez, Bandettini, \& Ungerleider, 2002). Our objective here was to investigate its use in a demanding cognitive task so as to further describe the viability of this analysis strategy when fMRI signals are employed - which are typically viewed as offering considerably less specific information relative to spike data, for instance.

In the present study, subjects performed a stop signal task in which they were required to withhold responding upon hearing an auditory cue among a rapid stream of visual go trials (Figure 2). Because such stop trials were infrequent and spaced apart in time, trial-by-trial behavioral outcome - namely, successful versus unsuccessful inhibition - was linked to single-trial response amplitude. Typically, previous neuroimaging studies of the stop signal task have employed fast event-related designs and have reported that mean responses between successful and unsuccessful stop trials differ in the extent to which they engage the right IFC and various other regions implicated in response inhibition. By using trialbased analysis, we aimed to further characterize the relationship between fMRI responses and observed behavior and, in particular, to test whether parametric increases in response amplitude could be quantitatively linked to the probability of successful response inhibition. More generally, quantifying the strength of this relationship allows one to compare the predictive power across different brain regions that are engaged during response inhibition. In this manner, a more complete characterization of the extent to which specific regions contribute to successful task performance is provided. Furthermore, research studies comparing inhibitory performance between groups (e.g., ADHD vs. controls) or between conditions within the same subjects (e.g., reward vs. no reward [Padmala \& Pessoa, 2010]) can employ trial-based analysis to quantify potential changes in the relationship between moment-to-moment fluctuations in fMRI responses and behavior - which can be done at both the group and individual levels.

A second goal of the present study was to investigate functional interactions between brain regions during response inhibition (Duann et al., 2009). We reasoned that because successful performance during response inhibition depends on the effective allocation of attention, the relationship (or coupling) between responses in attentional regions in the frontoparietal cortex (Corbetta \& Shulman, 2002; Kastner \& Ungerleider, 2001) and responses in regions more directly implicated in response inhibition (such as the right IFC) would vary as a function of behavioral performance - namely, successful versus unsuccessful inhibition. We therefore sought to investigate interregional signal relationships and their link to behavior on a trial-by-trial basis. For instance, if responses in the right IFC are predictive of successful performance, how is this relationship dependent onnamely, moderated by-fluctuations in responses in brain regions important for attention? In general, because network interactions are believed to subserve behavioral performance, developing trial-by-trial analyses to include signals from multiple regions will, we contend, prove to be an invaluable tool in characterizing the brain bases of behavior (Figure 1B).

\section{METHOD}

\section{Subjects}

Thirty-five volunteers ( $22 \pm 3$ years old; 19 females) participated in the study, which was approved by the Institutional Review Board of Indiana University, Bloomington. All the subjects were in good health, with no past history of psychiatric or neurological disease, and had normal or corrected-to-normal vision. All the subjects gave informed written consent. One subject's data were removed from the analysis because of unusually poor performance ( $70 \%$ correct on go trials).

\section{Stimuli and Behavioral Task}

We employed a stop signal task to investigate the neural correlates of response inhibition (Figure 2). We used a simple choice reaction time (RT) task, which included both go and stop trials. Each go trial started with the presentation of a simple shape stimulus, and the subjects were asked to indicate circle or square via a buttonpress on an MR-compatible response box by using the index or middle finger of their right hand. The subjects were instructed to respond as soon as possible during the presentation of the shape stimulus (trials with an RT longer than $1 \mathrm{sec}$ were treated as incorrect trials). Following the visual stimulus, the subjects viewed a blank screen for $1,000 \mathrm{msec}$. Stop trials were identical to go trials, except that a brief tone $(300 \mathrm{msec})$ was played after a variable stop signal delay (SSD) relative to the onset of the go stimulus, which indicated that the subjects should withhold their response (the initial value of the SSD was set to $250 \mathrm{msec}$ ). The SSD was adjusted dynamically throughout the experiment, such that if the subjects successfully inhibited their response on a stop trial, the SSD was increased by $50 \mathrm{msec}$ on a subsequent stop trial, and if the subjects failed to inhibit their response, the SSD was reduced by $50 \mathrm{msec}$ on a subsequent stop 
A
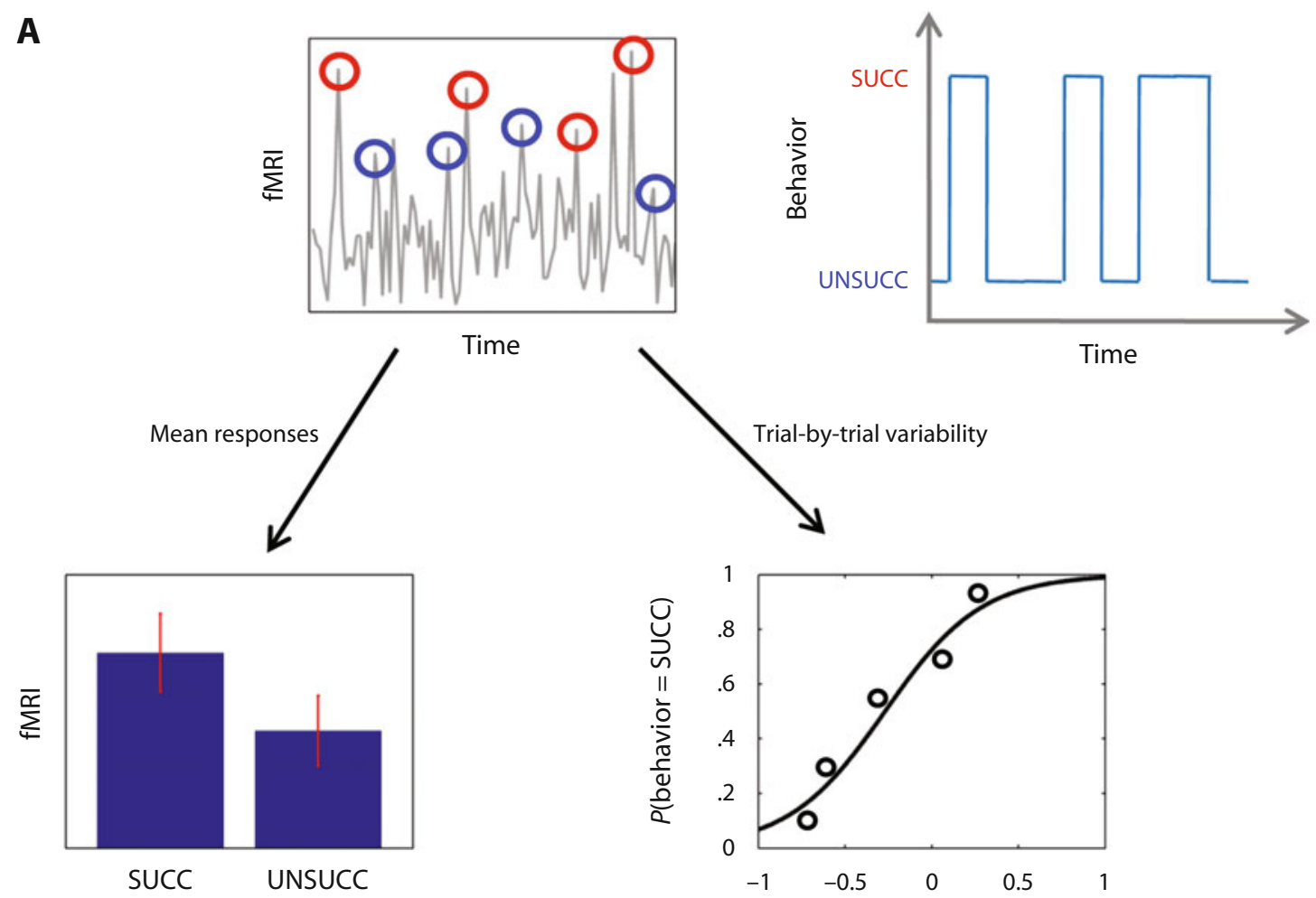

Time

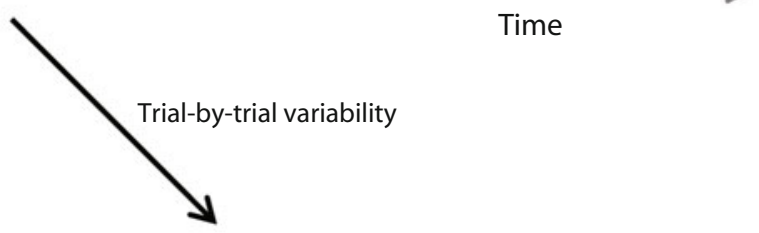

- Difference in means

$\cdot t$ statistic

B

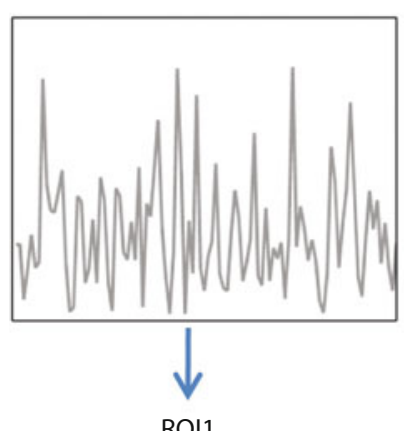

ROI1

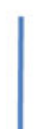

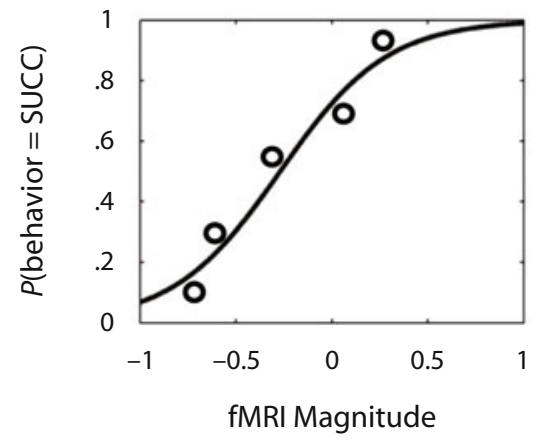

- Probability of behavior

- Logistic slope
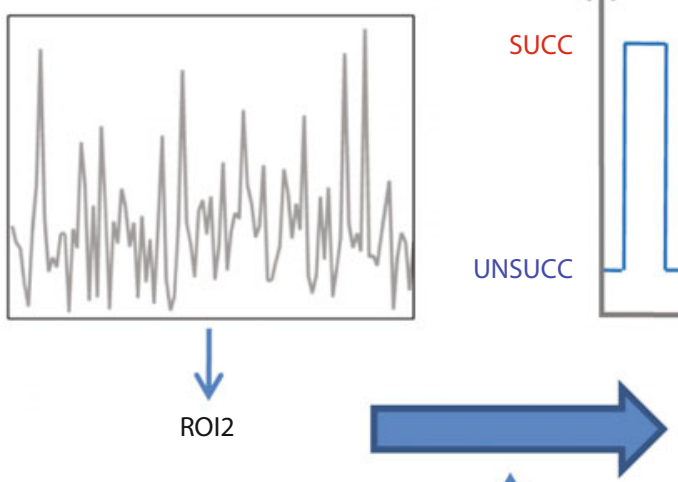

UNSUCC

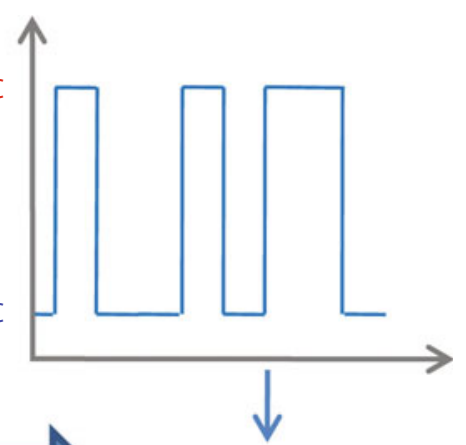

Behavior

Figure 1. Overall logic of trial-by-trial analysis. (A) The top row illustrates a schematic fMRI time series, with responses during successful and unsuccessful trials indicated in red (gray) and blue (black), respectively. The right panel shows a schematic representation of the behavior. If critical trials (stop trials, in the present case) are sufficiently spaced apart, the trial-by-trial variability in the magnitude of the evoked response can be quantitatively linked to the probability of successful performance via logistic regression analysis (bottom right). This analysis complements more standard ones based on mean responses (bottom left). (B) Trial-by-trial analysis can also take into consideration signals from multiple regions. For example, the link between single-trial responses in ROI2 and behavior can be evaluated as a function of the signal strength in ROI1 to assess the presence of a moderation-like pattern. In this case, the slope of the ROI2-to-behavior relationship (as in panel A) would depend on the strength of the evoked responses in ROI1. SUCC, successful stop trial; UNSUCC, unsuccessful stop trial. 


\section{Stop Signal Task}

Go Trials (84\%)

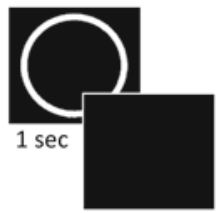

$1 \mathrm{sec}$

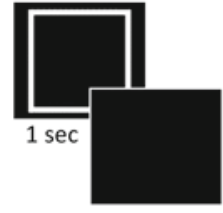

$1 \mathrm{sec}$ Stop Trials (16\%)

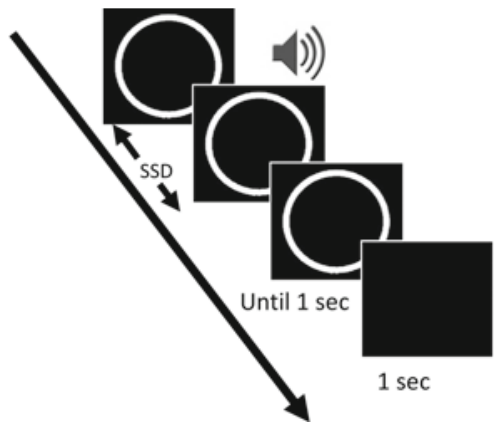

Figure 2. Stop signal task paradigm. During go trials, subjects responded to the go signal (circle or square?), whereas during stop trials, they were instructed to withhold their motor response (signaled by an auditory cue). The stop signal followed the go stimulus after a variable-length delay, the stop signal delay (SSD), which was updated on the basis of a staircase procedure that maintained behavioral performance at approximately $\mathbf{5 0 \%}$ correct.

trial (Logan, Schachar, \& Tannock, 1997; Rubia et al., 2003). This staircasing procedure ensured that the subjects were successfully inhibiting their responses on approximately $50 \%$ of the stop trials. The subjects were instructed to respond as quickly and accurately as possible and were asked to inhibit their response upon hearing a tone that followed the initial shape stimulus. They were also told that sometimes it might not be possible to successfully inhibit their response and that, in such cases, they should simply continue performing the task. Overall, the importance of going and stopping was stressed equally. The subjects performed a short practice run (approximately $4 \mathrm{~min}$ ) during the initial anatomical scan (see below) to familiarize themselves with the task.

Each subject performed four runs. Each run contained a total of 150 trials, out of which there were $126(84 \%)$ go trials and $24(16 \%)$ stop trials. Trial order was randomized but fixed across subjects; no stop trials occurred during the last 6 trials of each run. Go and stop trials contained circle and square shape stimuli in equal proportion. The data from a second condition (presented in separate experimental runs) involving reward were not analyzed here and will be discussed elsewhere. The runs from this second condition were identical in length to the runs reported here and alternated with the main ones (the order was counterbalanced across subjects).

Because standard slow event-related designs would be problematic in the context of fast-paced stop signal tasks and given that the primary interest of this study was to perform a trial-by-trial analysis, approximately $75 \%$ of the stop trials (i.e., 72 out of 96 trials) were spaced apart by at least $10 \mathrm{sec}$ from the other stop trials (with a minimum of 5 go trials in between) to allow for the estimation of single-trial responses (considering go trials as baseline condition; see below). The remaining stop trials were presented in close temporal succession, so as to prevent the subjects' expectancies from developing, but were not included in the trial-by-trial analysis.

\section{MR Data Acquisition}

MR data were collected using a 3 Tesla Siemens TRIO scanner (Siemens Medical Systems, Erlangen, Germany). Each scanning session began with a high-resolution MPRAGE anatomical scan ( $\mathrm{TR}=1,900 \mathrm{msec}, \mathrm{TE}=4.15 \mathrm{msec}$, TI $=1,100 \mathrm{msec}, 1-\mathrm{mm}$ isotropic voxels, 256-mm field of view). Subsequently, in each functional run, 153 volumes were acquired with a TR of 2,000 msec and a TE of $25 \mathrm{msec}$ and consisted of 34 axial slices with a thickness of $3.8 \mathrm{~mm}$ and an in-plane resolution of $3.8 \times 3.8 \mathrm{~mm}(240-\mathrm{mm}$ field of view).

\section{Behavioral Data Analysis}

As was stated above, the SSD was adjusted dynamically to yield an inhibition success rate of approximately $50 \%$. The stop signal RT (SSRT), which provides an estimate of the inhibitory RT, was calculated by subtracting the average SSD from the median RT during correct go trials, following the race model (Logan \& Cowan, 1984).

\section{General fMRI Data Analysis}

Preprocessing of the data was done using tools from the AFNI software package (Cox, 1996) (http://afni.nimh.nih.gov/afni). The first three volumes of each functional run were discarded to account for equilibration effects. The remaining volumes were slice-time corrected and spatially registered for motion correction to the volume acquired closest in time to the particular subject's high-resolution anatomy. The functional data were then normalized to Talairach space (Talairach \& Tournoux, 1988) and spatially smoothed using a Gaussian filter with a full-width at half maximum of $7.6 \mathrm{~mm}$ (i.e., two times the voxel dimension). Finally, the signal intensity of each voxel was scaled to a mean of 100 .

\section{Voxelwise Analysis}

Voxelwise analyses were run to determine regions of interest (ROIs; see below). Each subject's fMRI data were analyzed using standard multiple linear regression. A linear model was defined for each subject that included three regressors corresponding to the three main event types - namely, successful stop trials (SUCC), unsuccessful stop trials (UNSUCC), and an event type that included all incorrect go trials (INC). All regressors were convolved with a canonical hemodynamic response function (Cohen, 1997) to account for the low-pass properties of fMRI responses. Constant, linear, and quadratic terms were included for each run separately (as covariates of no interest) to model drifts of the MR signal. Correct go trials were not modeled explicitly and constituted the implicit baseline in the model. This type of baseline condition has been used successfully in several fMRI studies of the stop signal task (Chamberlain et al., 2009; Rubia et al., 2003; Rubia, Smith, Taylor, \& Brammer, 2007). In this manner, all parameter estimates reported in this study should be interpreted relative to the responses evoked by correct go trials.

\section{ROI Analysis}

To maximize statistical power, we focused our analysis on a set of ROIs that have been reported consistently in the previous re- 
sponse inhibition literature (as listed in Table 2); note, in particular, that regions such as the posterior cingulate, anterior cingulate, and anterior insula have been implicated in, among other things, error-related processing during these tasks. Specifically, the precise location of these ROIs was determined on the basis of general taskrelated activation, formally defined by the contrast vector $\boldsymbol{c}_{\text {selection }}=$ $\left[\begin{array}{lll}1 & 1 & 0\end{array}\right]^{\mathrm{T}}$ (where $\mathrm{T}$ denotes the transpose operation), corresponding to the SUCC, UNSUCC, and INC conditions, respectively, at a $p$ value of .005 , corrected for multiple comparisons according to a false discovery rate procedure (Genovese, Lazar, \& Nichols, 2002). Individual ROIs were drawn using a sphere of 5-mm radius centered at the peak voxel of each cluster (defined at the group level). For each individual, a representative time series for the ROI was then defined by averaging across all of the voxels. Before doing so, the variance explained by incorrect go trials was removed from a voxel's time series (slow-varying drifts in MR signal were likewise removed). A convenient way to implement this procedure is available via the $3 \mathrm{dSynthesize}$ program in AFNI. When our analyses were repeated without removing the variance related to incorrect go trials (which were fewer than $2.5 \%$ overall), nearly identical results were observed.

The selection criterion above was employed because it was orthogonal to the main contrast of interest in our study - namely, the contrast of successful and unsuccessful trials, specifically $c_{\text {test }}=$ $\left[\begin{array}{lll}1 & -1 & 0\end{array}\right]^{\mathrm{T}}$ (note that $\boldsymbol{c}_{\text {selection }}{ }^{\mathrm{T}} \boldsymbol{c}_{\text {test }}=0$ ). In this manner, the selection and test criteria were independent, avoiding potential biases in the ROI analysis. In addition, the staircasing procedure guaranteed that an approximately equal number of trials were obtained for each trial type of interest (Kriegeskorte, Simmons, Bellgowan, \& Baker, 2009).

We further simulated the effect of the ROI selection procedure by generating 500,000 Gaussian random samples $(M=0$, $S D=1$ ), with 36 trials per condition (the mean number of spaced apart trials per condition observed during the fMRI experiment). For every sample, we contrasted SUCC and UNSUCC trials via logistic regression analysis to generate the distribution of logistic regression slopes based on this noise distribution. These results were then compared with the distribution of slopes obtained when our selection criterion was first adopted (i.e., when the selection contrast was statistically significant). No selection bias was observed, as evidenced by the complete overlap of the two distributions (Supplemental Figure 1).

For illustration of average evoked responses for the SUCC and UNSUCC conditions, for each ROI (see Figures 3-6), selective averaging was performed using responses to stop trials that were spaced at least $10 \mathrm{sec}$ apart, as in the trial-based analysis (see below).

\section{Trial-Based Analysis}

To quantify the link between fMRI amplitude and behavior at the individual level, a trial-by-trial fMRI analysis was performed on the time series from each ROI for each individual. We modeled the probability of success during a stop trial by performing standard logistic regression analysis (Hosmer \& Lemeshow, 2000) based on single-trial fMRI response amplitude. Because the SSD varied for each trial, due to the staircasing procedure, these values were also entered into our model, together with the interaction between SSD and fMRI amplitude (to avoid strong correlations between the interaction and remaining terms, the fMRI and SSD variables were initially mean corrected). Thus, the probability of success was modeled by

$\operatorname{Pr}\left(y_{i}=1\right)=\operatorname{logit}^{-1}\left[b_{0}+b_{1} \mathrm{fMRI}_{i}+b_{2} \mathrm{SSD}_{i}+b_{3}\left(\mathrm{SSD}_{i} \times \mathrm{fMRI}_{i}\right)\right]$,

where $y$ is the behavioral outcome (success, 1 ; failure, 0 ), the function logit ${ }^{-1}$ transforms continuous values to the range $(0,1)$, which is necessary for probabilities (note that this is simply a logistic sigmoidal curve), fMRI is the response amplitude, SSD is the staircasing delay, and $i$ is a trial index. For plotting purposes (see Figures 3-6), only the influence of fMRI responses is illustrated (thus keeping our plot two-dimensional). In the plots of individual data fits (Figures 3-6, panels A and E), data were binned into 14 bins based on fMRI amplitude such that 5 trials were present in each bin (except the last one, which had 7 trials); data points represent the proportion of SUCC trials in each bin. A similar strategy was used for group fits (Figures 3-6, panels C and G), but in this case, data were pooled across subjects and 24 bins were used with approximately 100 trials in each bin (see below for the methods employed in the group fits). Finally, note that whereas a linear regression fit to the data is conceivable (see, e.g., Figure $3 \mathrm{C}$ ), a logistic-type fit is required given the binary nature of the data (success/failure).

Response strength for each trial was indexed by considering activations around the expected peak time - specifically, the time points at 4,6 , and $8 \mathrm{sec}$ following trial onset (only trials sufficiently spaced apart were considered). Because responses were somewhat variable in their timing (see Figures 3-6), the peak response during the 4- to 8 -sec window was averaged with the second-largest response in the same window (only consecutive points were averaged). For instance, if the peak occurred at $6 \mathrm{sec}$ and the second-largest response was at $4 \mathrm{sec}$, responses at 4 and $6 \mathrm{sec}$ were averaged; if the second-largest response was at $8 \mathrm{sec}$, responses at 6 and $8 \mathrm{sec}$ were averaged. Critically, very similar results were obtained when only the peak response between 4 and $8 \mathrm{sec}$ was used. For regions that exhibited deactivations relative to baseline (e.g., the left superior frontal gyrus), we averaged the two consecutive responses with the largest responses in absolute value (i.e., response minima).

To assess the link between single-trial amplitude and behavioral performance at the group level, a multilevel logistic regression analysis (Gelman \& Hill, 2007) was performed via the method of generalized estimating equations (GEE; Zeger \& Liang, 1986). GEE is a powerful statistical technique that is often used with longitudinal and correlated data, especially when the data are binary. GEE combines the information from all of the subjects and determines an overall population-level logistic function, taking into account the correlated structure of the data within subjects. As above, we modeled the probability of success during a stop trial as a function of response amplitude, SSD, and the interaction term. The slope of the logistic fit corresponding to response amplitude indicates the strength of the association between $\mathrm{fMRI}$ responses and behavior at a single-trial level (at the group level). To implement GEE, the GEEQBOX toolbox (Ratcliffe \& Shults, 2008) was used in MATLAB (The MathWorks, Natick, MA).

\section{Calculation of Average Predictive Comparisons on the Basis of $f$ MRI Amplitude}

Logistic regressions are nonlinear on the probability scale such that a constant fMRI signal difference does not correspond to a fixed change in probability; the gain will be greater at the middle portion of the sigmoidal curve where the slope is steepest. To provide a measure of the increase in probability of success as a function of fMRI signal changes, for each individual, we compared probabilities when activation changed from medium to high levels, where medium corresponded to the amplitude when $\operatorname{Pr}(y=$ success) $=.5$ and high corresponded to the mean response amplitude of the trials with the $10 \%$ highest responses. We defined high in this manner to minimize the effects of unusually high single-trial responses if the largest single trial (i.e., the max) were picked. Determining the predictive difference in probabilities between these two cases (medium and high) is admittedly arbitrary but provides an effective way to summarize how changes in fMRI responses affect the ability to predict successful performance (Gelman \& Hill, 2007). A complementary summary strategy is provided by the slope of the logistic fit at the group level, as evaluated via GEE (see above), a strategy that takes into account all trials (from all subjects). However, because the logistic fit represents a relationship that is nonlinear, the slope value is informative mostly about the region of greatest probability change (e.g., similar changes in 


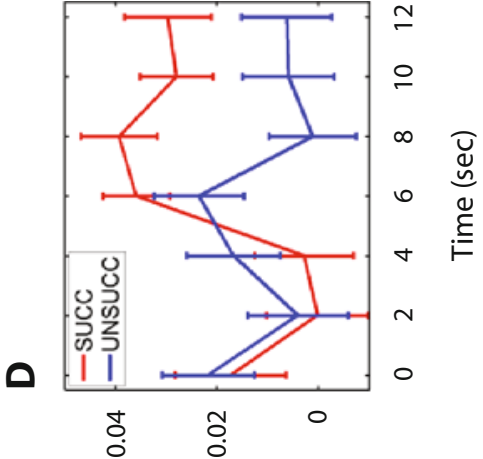

\% әбиечว ןеиб!s

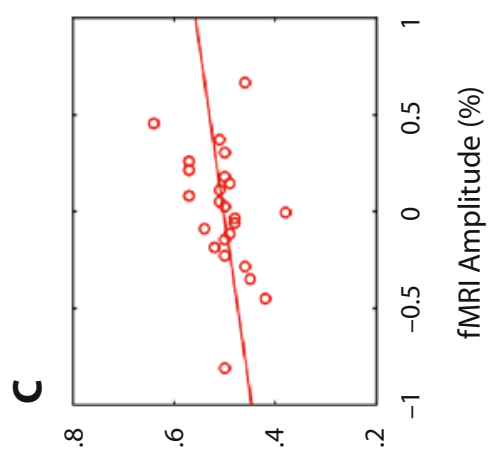

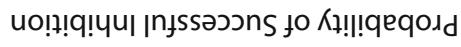

는

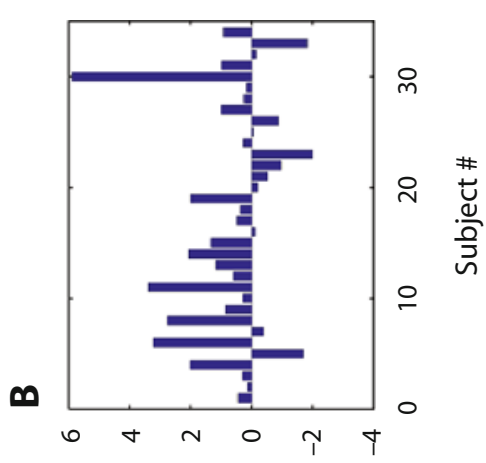

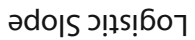

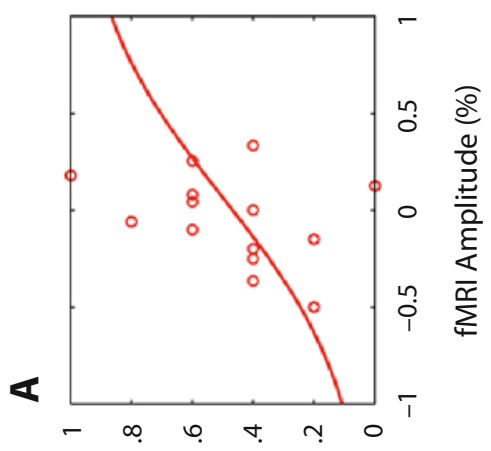

uo!+!q!yul jnfssəoכns fo Kł!!!qeqodd

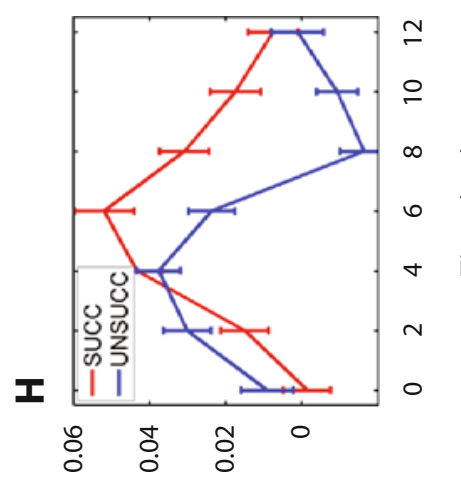

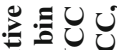

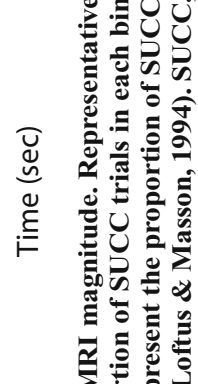

$\%$ әбиечว ןеиб!s
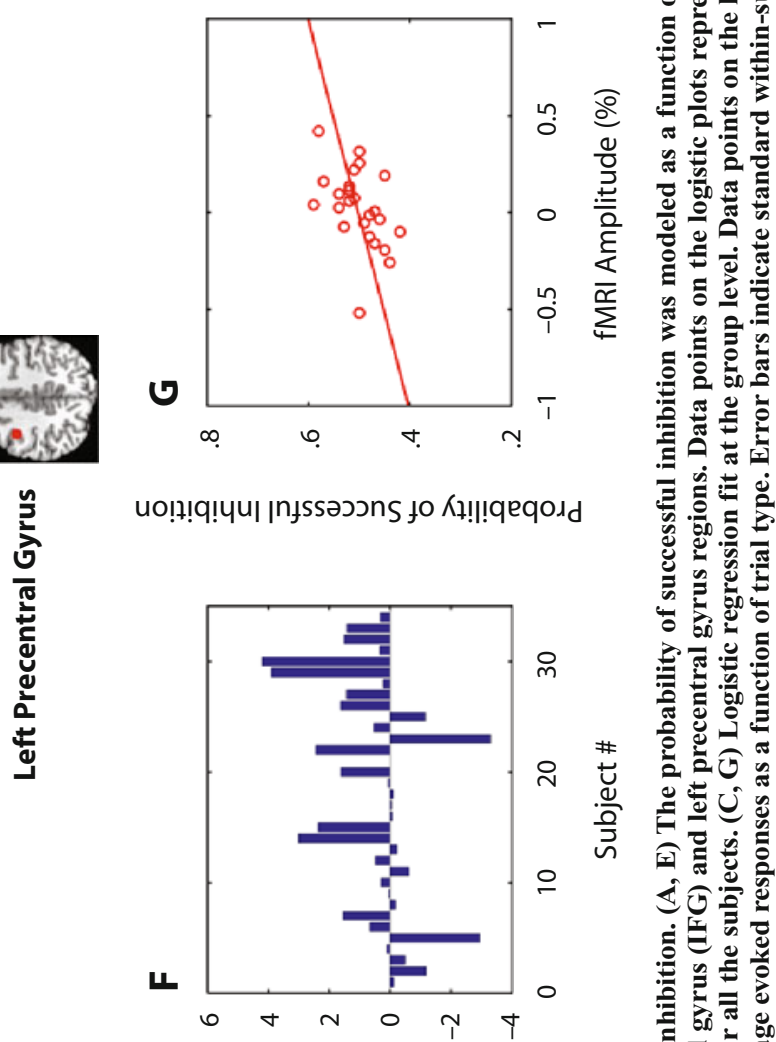

ədoㅇ ग!!s!

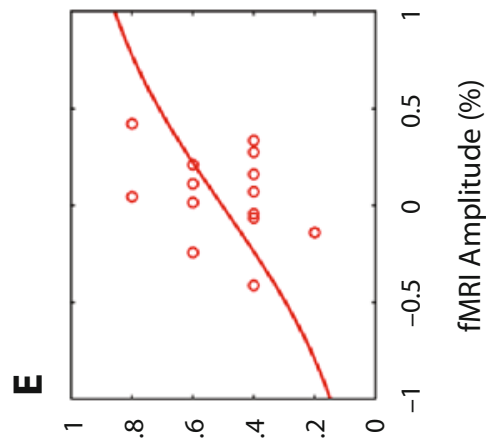

uo!!!q!Yul jnłssəoכns to Kł!!!qeqodd 


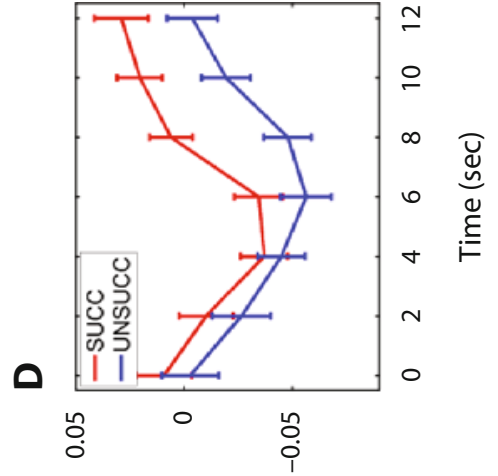

\% әбиечว ןеuб!s

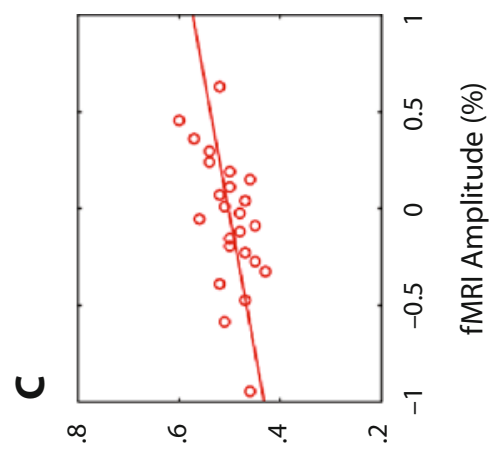

蓄

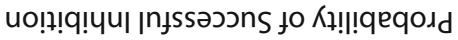

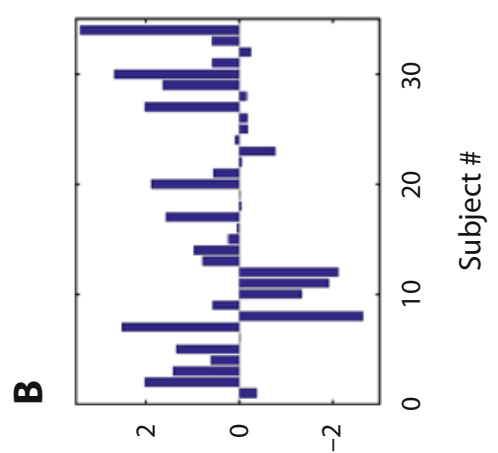

ədo|S ग!!s! 607

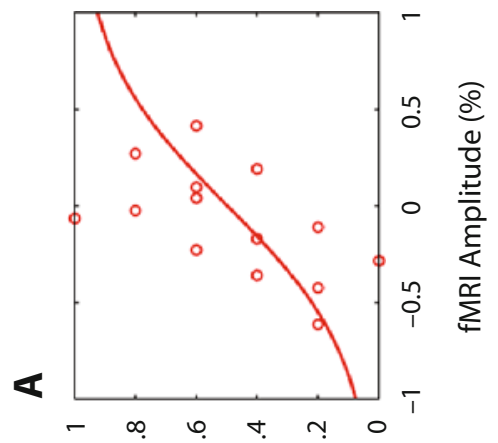

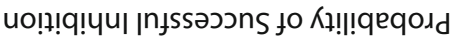

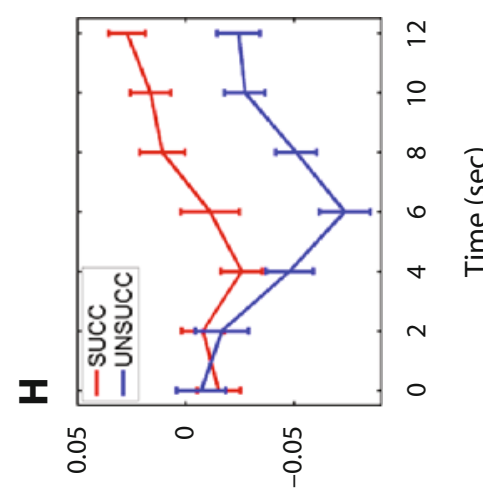

\% әбиечว |еиб!s
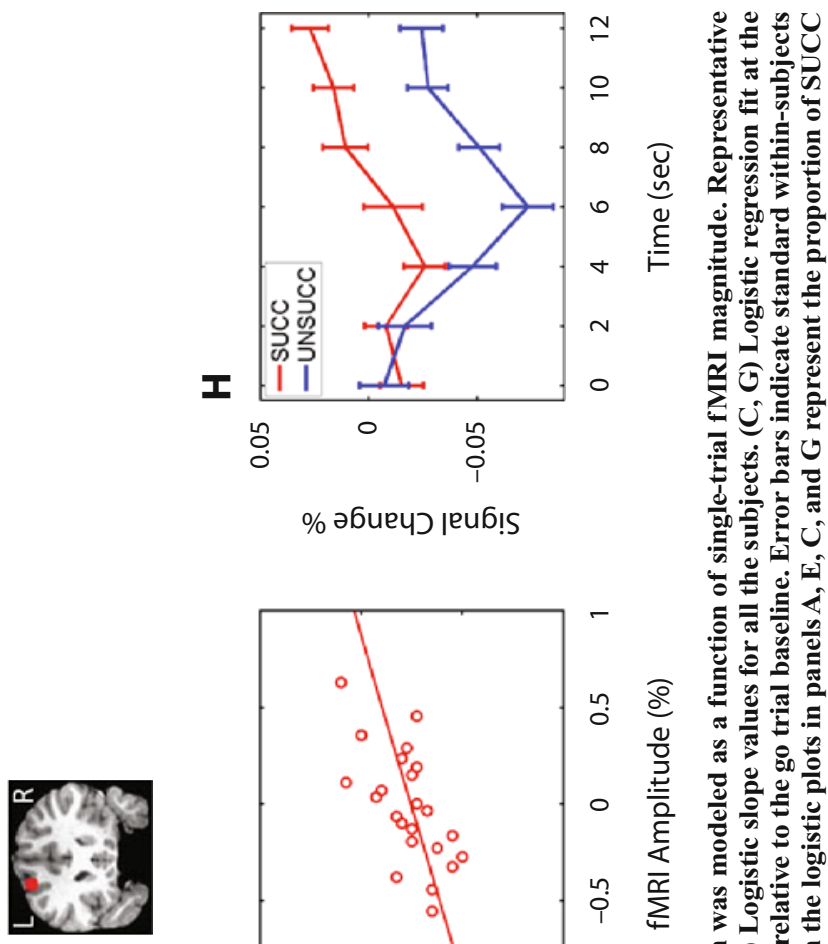

ํㅗㄴ

ᄂ

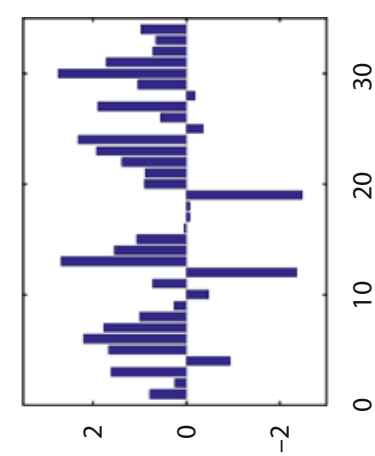

uo!!!q!yul |njssəoวns to Kl!!!qeqord

$\checkmark$

正它

象象

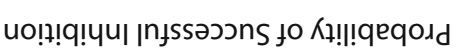

ədo|S ग!!s! 607

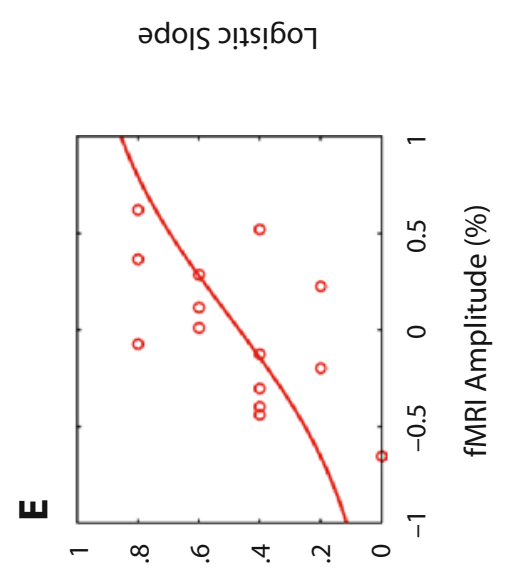

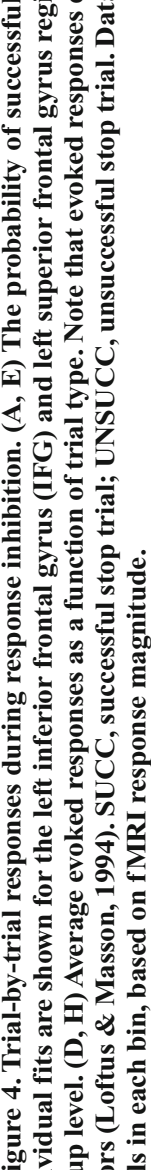




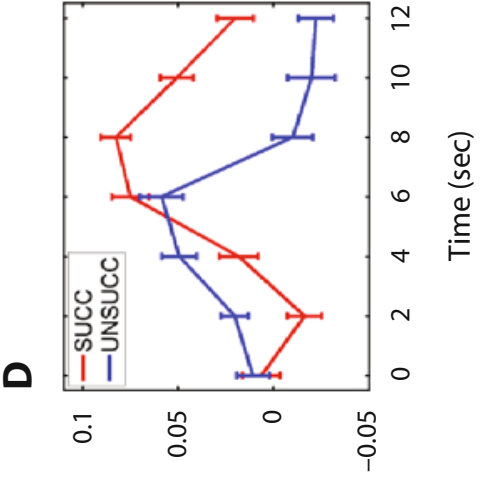

\% әбиечว ןеиб!s

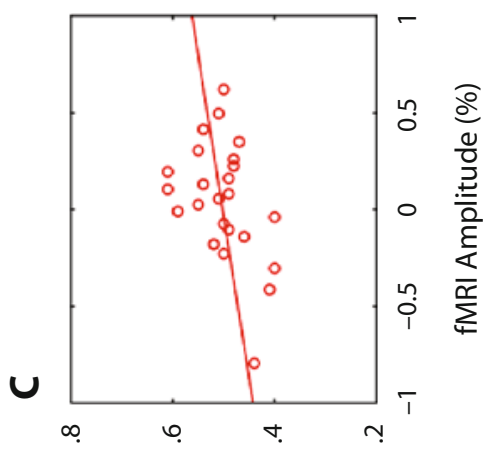

uo!?!q!yul jnfssəoวns to K!!!!qeqoגd
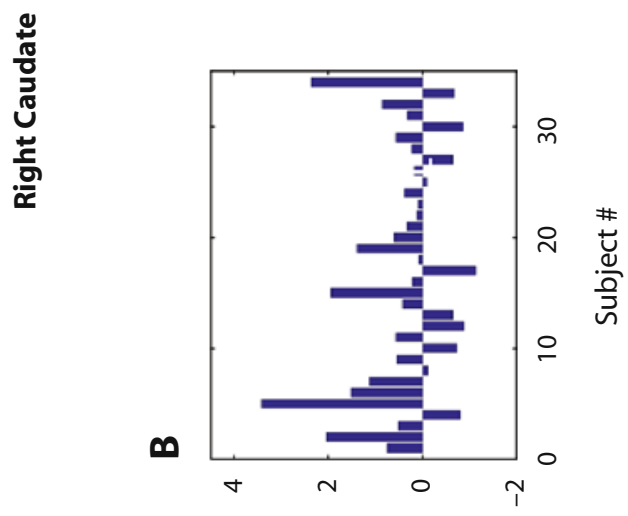

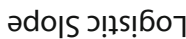

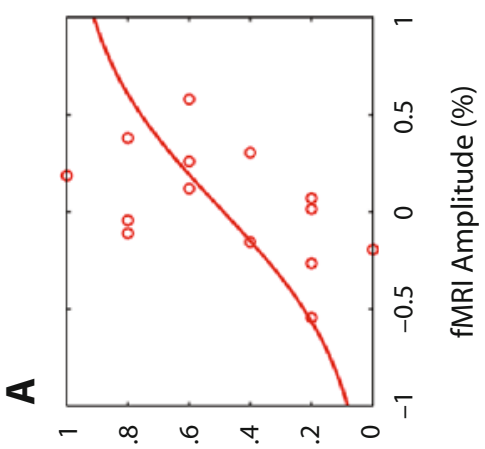

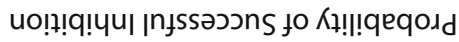

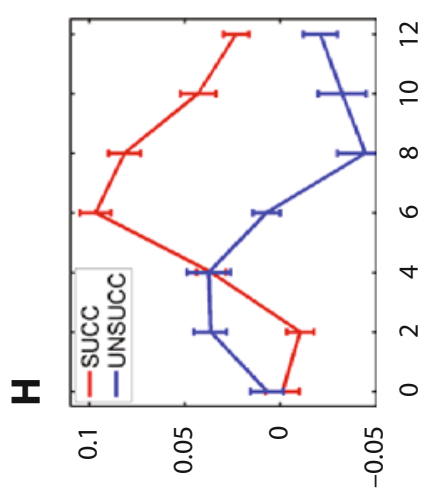

萿

跣

至

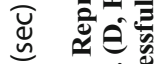

के

政

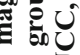

x.

\% әбиечว |еиб!s

要

$E$

कo

के

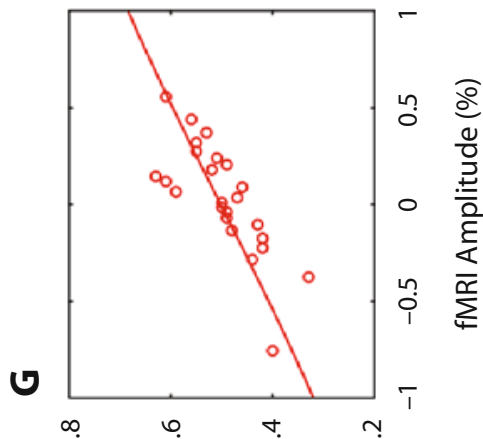

5.

政

J

๙ 0 U

¿

过

诤

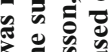

Е

एक

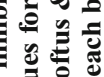

uo!!!q!บu |nıssəoכns to K!!!!qeqoגd

$\frac{5}{0}$
$\frac{0}{0}$
$\frac{3}{3}$
0
$\frac{0}{0}$
$\frac{0}{\alpha}$

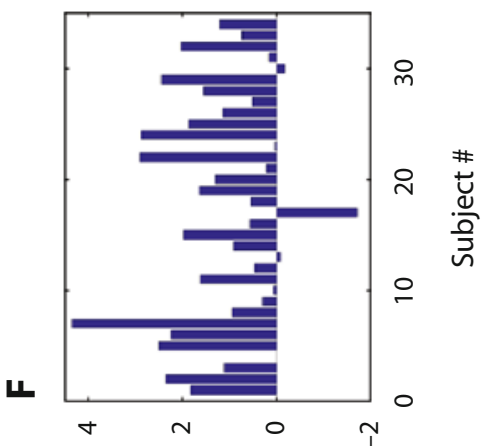

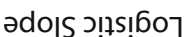

를 ․․ㄹ

造造

을

$\div$.

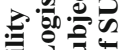

表全

들

造

ํํㄹ 을

(马)

结

휼

클

츨

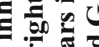

政

ถ

일 되되

일

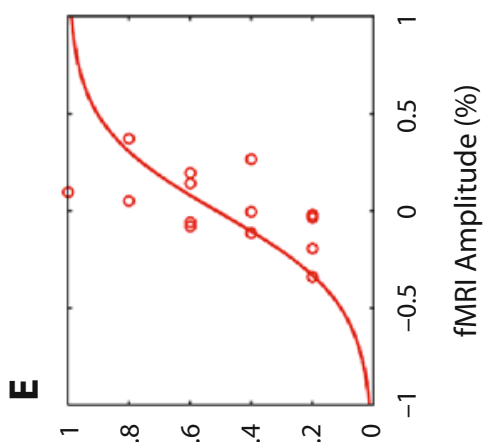

클

.

正

를

ㅎํ을

可资

政

군

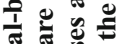

$\exists$ 气气 气

ن

를 


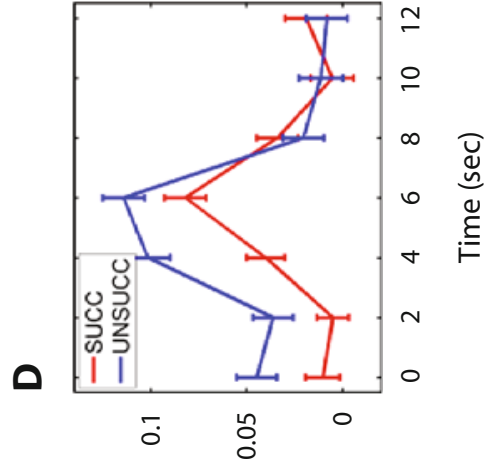

\% әбиечว ןеиб!s

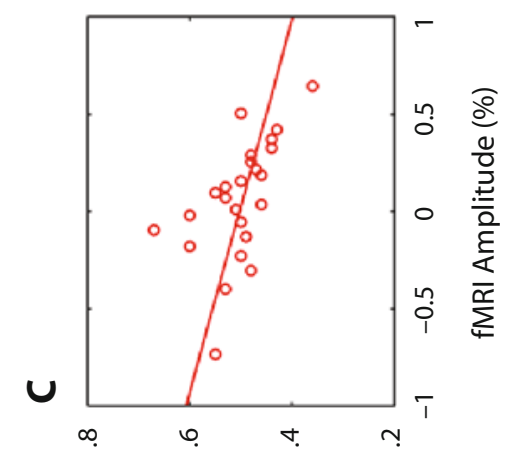

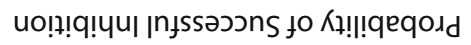

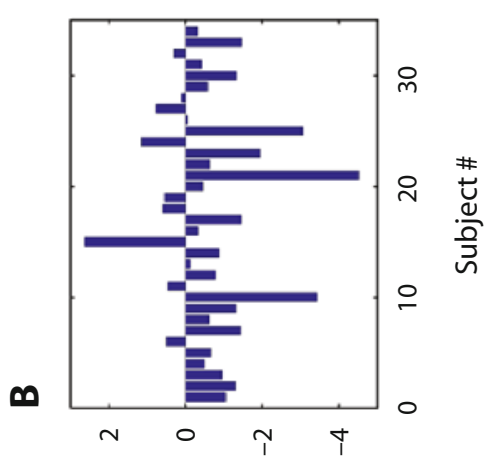

ədo|S ग!ฺs!60ך

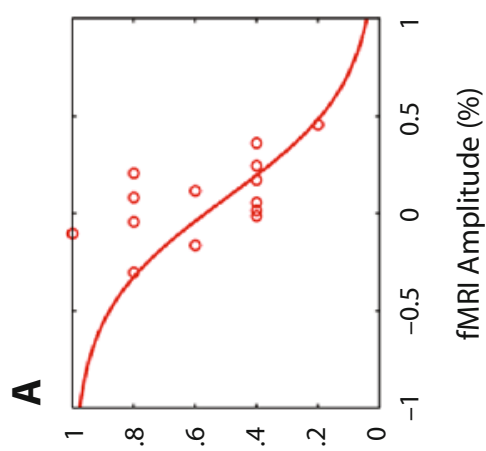

uo!!!q!yu |nfssəoวns to K!!!!qeqodd

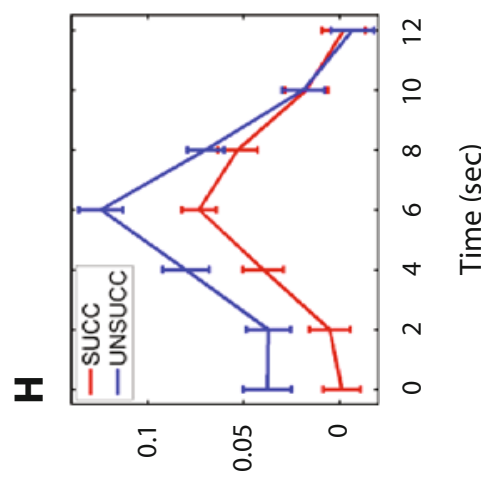

\% әбиечว ןеuб!s

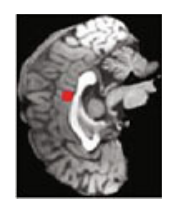

U

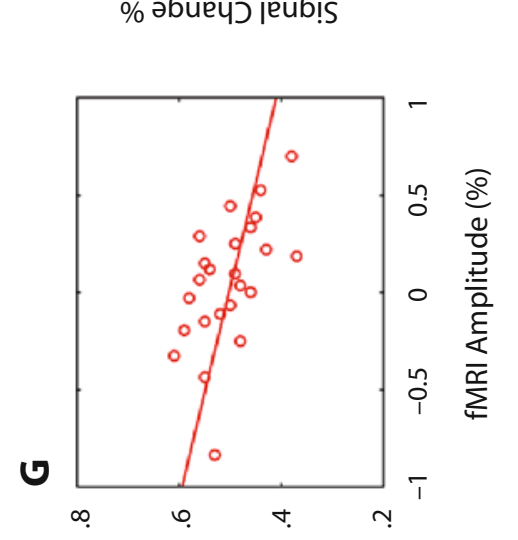

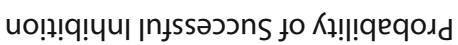

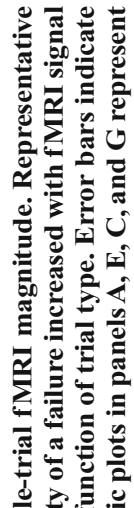

象政

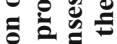

舟要

政

if

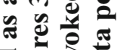

해유

일

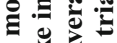

类

อิ่อ

率

old

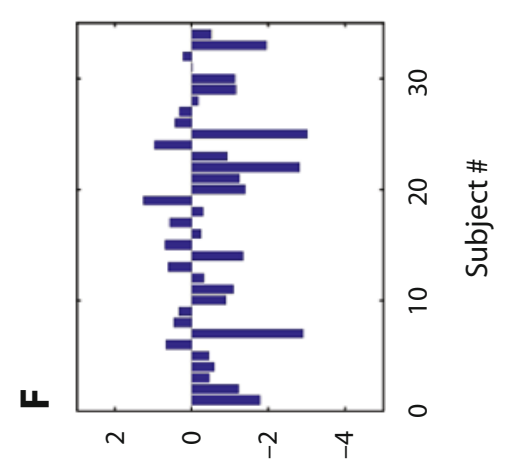

Sot $\frac{1}{5}$

节

$\overline{50}$

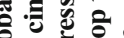

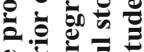

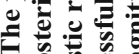

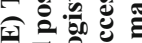

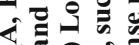

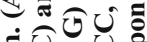

记记

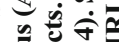

ədo|s ग!ฺธ!6oา

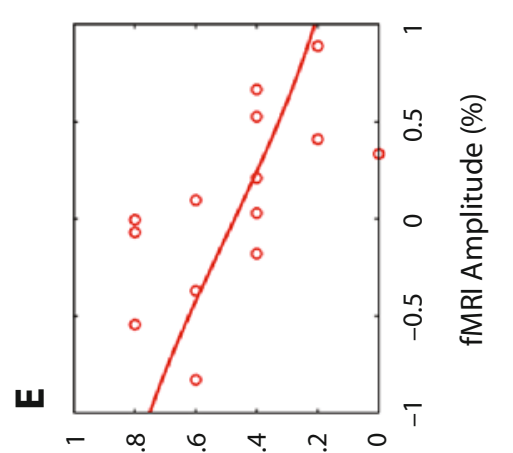

के के

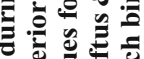

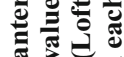

势

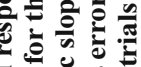

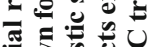

5

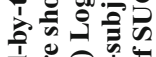

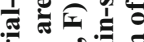

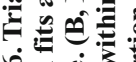

元$$
\begin{array}{llll}
- & 0 & 4 & 0
\end{array}
$$

uo!!!q!yul |njssəoכns to K?!!!qeqodd 
fMRI amplitude at low values of response amplitude do not change the probability of behavior by much).

More precisely, the average (across trials) predictive difference in probability for a given individual was given by $1 / n \Sigma \Delta_{i}$, where

$$
\begin{aligned}
\Delta_{i}= & \operatorname{logit}{ }^{-1}\left[b_{0}+b_{1} \text { high }+b_{2} \mathrm{SSD}_{i}+b_{3}\left(\mathrm{SSD}_{i} \times \text { high }\right)\right] \\
& -\operatorname{logit}^{-1}\left[b_{0}+b_{1} \text { medium }+b_{2} \mathrm{SSD}_{i}+b_{3}\left(\mathrm{SSD}_{i} \times \text { medium }\right)\right],
\end{aligned}
$$

$n$ is the number of trials, and $i$ is a trial index (all trials must be considered, because SSD varied as a function of trial; for further details, see Gelman \& Hill, 2007). This predictive difference in probability was computed for every subject and then averaged across subjects to provide a final value.

\section{Functional Connectivity Analysis}

We investigated functional interactions between attention- and inhibition-related regions. We use attention related and inhibition related as convenient shorthand terms, without implying that the associated regions are exclusively linked to these functions; in particular, the right IFC has been implicated in several executive functions (Brass, Derrfuss, Forstmann, \& von Cramon, 2005), including more reactive/exogenous attentional effects (Corbetta \& Shulman, 2002; Pessoa \& Ungerleider, 2004). On the basis of the existing literature, the following attentional regions were probed: the intraparietal sulcus (IPS; right, $x=28, y=-58, z=42$; left, $x=-29, y=-58, z=43$ ), the inferior parietal lobule (IPL; right, $x=36, y=-37, z=44$; left, $x=-34, y=-37, z=38$ ), and the frontal eye field (FEF; right, $x=28, y=-8, z=51$; left, $x=$ $-24, y=-13, z=54)$. For these regions, ROIs were defined as described previously - namely, by assessing general task-related activation and by creating representative time series. Likewise, the following inhibition regions were probed: the right inferior frontal gyrus (IFG), bilateral caudate, and bilateral putamen (we did not include the left IFG because it exhibited a different response pattern, as compared with the preceding regions; see Figure 4D).

Initially, we investigated whether the correlations between trialby-trial responses in inhibition-related regions were more strongly correlated with signals in frontoparietal attentional regions during SUCC versus UNSUCC trials. Prior to computing the correlations, response strengths were mean centered, such that only deviations from the mean were considered. Note also that because we employed a slow event-related design, the correlations involved a single measure of response strength per trial (i.e., the correlations did not involve the entire time series; see Zhou, Thompson, \& Siegle, 2009). To compare the difference in correlations between the SUCC and UNSUCC conditions at the group level, correlations were initially transformed (via Fisher's $Z$ transform) and then compared via a paired $t$ test.

\section{Relationship Between Frontoparietal Responses and the Strength of the Relationship Between the Right IFG and Behavior}

We investigated the relationship between trial-by-trial fluctuations in responses in attentional regions (IPS, IPL, and FEF) and the strength of the association between right IFG and behavior. To do so, we probed whether the trial-by-trial relationship between the right IFG and behavior depended on the magnitude of the IPS, IPL, or FEF (each tested in a separate analysis). To increase statistical power, we pooled data from all the subjects and binned trials on the basis of the strength of frontoparietal activation independent of behavioral responses. For each bin, all the trials were employed to determine the logistic regression slope between right-IFG response strength and behavior (successful vs. unsuccessful). The regression slopes were then correlated with the median amplitude of frontoparietal responses in each bin (see Figure 10B for further details). A total of 22 bins was used to partition the range of frontoparietal responses into approximately equal number of trials per bin. Note that the results were robust with respect to the specific number of bins employed when we partitioned the data into 10-25 bins.
Table 1

Behavioral Results

Median go reaction time $(\mathrm{msec})$

$487.1 \pm 18.6$

Inhibition rate $(\%)$

Stop signal delay ( $\mathrm{msec}$ )

Stop signal reaction time ( $\mathrm{msec})$

$50.3 \pm 0.7$

$282.4 \pm 25.1$

Unsuccessful stop trial reaction time $(\mathrm{msec}) \quad 462.7 \pm 17.5$

Go error rate (\%)

\section{RESULTS}

\section{Behavioral Results}

Behavioral results are summarized in Table 1 (the results are for the same set of trials as that used in the fMRI analysis; see the Method section). Mean RT on correct go trials was $487 \mathrm{msec}$, and the mean go trial error rate was $2.3 \%$. As was expected, because of the staircasing procedure, the inhibition rate during stop trials was approximately $50 \%$. The critical behavioral index of response inhibition, SSRT, was on average $205 \mathrm{msec}$, in the range previously reported in studies of the stop signal task (Colzato, van den Wildenberg, \& Hommel, 2007; Williams, Ponesse, Schachar, Logan, \& Tannock, 1999). Finally, the RT during UNSUCC trials was shorter than those on correct go trials $[t(33)=5.74, p<.001]$, in line with predictions of the race model (Logan \& Cowan, 1984).

\section{fMRI Results}

To maximize statistical power, trial-by-trial analyses were restricted to a set of ROIs (Table 2; see the Method section). For each ROI, we investigated the link between single-trial amplitude and behavioral performance at the individual level by performing logistic regression analysis. Accordingly, fMRI response amplitude was employed to predict whether the subjects were successful at withholding responses during stop trials. Positive slopes indicate that the probability of successful inhibition during a stop trial increased as a function of the amplitude of the evoked response. Examples of logistic fits at the individual level are shown in Figure 3 for the right IFG (panel A) and left precentral gyrus (panel E), together with bar plots showing slope values for all the subjects (panels B and F). Group-level logistic fits are shown in the third column (panels $\mathrm{C}$ and $\mathrm{G}$; note that for the range of fMRI amplitudes considered, the figure illustrates the linear part of the logistic function). Finally, mean evoked responses are shown in the last column (panels D and $\mathrm{H}$ ).

Both the right IFG and the left precentral gyrus have been advanced as important regions for response inhibition. In this context, the results of Figure 3 can be summarized as follows. In both of these regions, mean evoked responses were stronger during SUCC than during UNSUCC trials. Critically, as the single-trial response amplitude increased, the probability of successfully inhibiting a motor response increased. This relationship was observed at the level of the individual for most subjects (in terms of the sign of the slope) and reliably at the group level. Note that even though a small number of trials were employed 
Table 2

Region of Interest (ROI) Based Trial-by-Trial Logistic Analysis Linking fMRI Signals to Behavior (Peak Talairach Coordinates Are Provided for Each ROI)

\begin{tabular}{|c|c|c|c|c|c|c|c|}
\hline \multicolumn{5}{|c|}{ Location } & \multirow[b]{2}{*}{ fMRI Slope } & \multirow{2}{*}{$\begin{array}{c}\mathrm{fMRI} \times \mathrm{SSD} \\
\text { Slope }\end{array}$} & \multirow[b]{2}{*}{$\operatorname{UNSUCC}(t)^{*}$} \\
\hline & & $x$ & $y$ & $z$ & & & \\
\hline \multicolumn{8}{|c|}{ SUCC $>$ UNSUCC } \\
\hline \multicolumn{8}{|l|}{ Parietal } \\
\hline Intraparietal sulcus & $\mathrm{R}$ & 28 & -58 & 42 & $\mathbf{0 . 3 3}(p<.05)$ & $-2.45(p=.20)$ & $3.47(p<.005)$ \\
\hline Inferior parietal lobule & $\mathrm{R}$ & 36 & -37 & 44 & $0.89(p<.005)$ & $0.20(p=.94)$ & $1.08(p=.29)$ \\
\hline \multicolumn{8}{|l|}{ Frontal } \\
\hline \multirow[t]{2}{*}{ Inferior frontal gyrus } & $\mathrm{R}$ & 48 & 26 & 20 & $\mathbf{0 . 2 3}(p<.05)$ & $-2.14(p=.16)$ & $1.10(p=.28)$ \\
\hline & $\mathrm{L}$ & -48 & 26 & 14 & $\mathbf{0 . 2 8}(p<.05)$ & $-1.74(p=.14)$ & $-3.68(p<.005)$ \\
\hline Superior frontal gyrus & $\mathrm{L}$ & -19 & 19 & 49 & $0.46(p<.005)$ & $2.18(p=.14)$ & $-4.64(p<.001)$ \\
\hline Precentral gyrus & $\mathrm{L}$ & -34 & -11 & 45 & $\mathbf{0 . 4 0}(p<.05)$ & $-1.01(p=.71)$ & $4.91(p<.001)$ \\
\hline Supplementary motor area & $\mathrm{R} / \mathrm{L}$ & 0 & -4 & 52 & $-0.06(p=.65)$ & $-2.51(p=.11)$ & $7.37(p<.001)$ \\
\hline \multicolumn{8}{|l|}{ Subcortical } \\
\hline \multirow[t]{2}{*}{ Putamen } & $\mathrm{R}$ & 21 & 4 & 4 & $0.76(p<.005)$ & $-0.50(p=.80)$ & $2.51(p<.010)$ \\
\hline & $\mathrm{L}$ & -19 & 4 & 4 & $0.68(p<.005)$ & $-0.09(p=.96)$ & $2.03(p<.050)$ \\
\hline \multirow[t]{2}{*}{ Caudate } & $\mathrm{R}$ & 11 & 4 & 9 & $0.24(p<.05)$ & $-0.36(p=.85)$ & $4.62(p<.001)$ \\
\hline & $\mathrm{L}$ & -10 & 4 & 8 & $0.29(p<.05)$ & $-0.12(p=.94)$ & $4.22(p<.001)$ \\
\hline Subthalamic nucleus & $\mathrm{R}$ & 10 & -14 & -3 & $0.04(p=.80)$ & $-0.03(p=.99)$ & $3.46(p<.005)$ \\
\hline \multicolumn{8}{|c|}{ UNSUCC > SUCC } \\
\hline Posterior cingulate & $\mathrm{R} / \mathrm{L}$ & 0 & -25 & 28 & $-\mathbf{0 . 3 7}(p<.005)$ & $-1.84(p=.26)$ & $8.46(p<.001)$ \\
\hline Anterior cingulate & $\mathrm{R} / \mathrm{L}$ & 0 & 20 & 28 & $\mathbf{- 0 . 4 2}(p<.005)$ & $\mathbf{- 3 . 2 4}(p<.05)$ & $8.64(p<.001)$ \\
\hline \multirow[t]{2}{*}{ Anterior insula } & $\mathrm{R}$ & 35 & 14 & 6 & $-0.23(p=.22)$ & $\mathbf{- 5 . 3 7}(p<.01)$ & $13.88(p<.001)$ \\
\hline & $\mathrm{L}$ & -29 & 17 & 7 & $-0.20(p=.23)$ & $-\mathbf{5 . 6 3}(p<.01)$ & $13.96(p<.001)$ \\
\hline
\end{tabular}

Note-SSD, stop signal delay; SUCC, successful inhibition; UNSUCC, unsuccessful inhibition. * *Standard contrast between unsuccessful trials and baseline (as suggested by a reviewer).

per subject, the fit was significant at the level of the individual for some of them.

Positive logistic slopes at the individual and group levels were also observed in two other PFC regions - namely, the left IFG and left superior frontal gyrus (Figure 4). Unexpectedly, however, mean responses exhibited a different pattern when compared with those shown in Figure 3. Specifically, both SUCC and UNSUCC trials displayed decreased activation relative to baseline (go trials).

Positive logistic slopes were also observed in the right IPL and right IPS (see Table 2 for complete results). Furthermore, significant group-level positive slopes were observed in subcortical regions, including the caudate and putamen (both bilaterally), as illustrated in Figure 5.
It is of interest to consider the slope values for the regions discussed in the preceding paragraphs. Overall, the slope varied more than threefold, with the lowest values being observed in the right IFG (.23) and right caudate (.24) and the highest values being observed in the right putamen (.76) and right IPL (.89).

Interestingly, some regions exhibited significant grouplevel slopes that were negative, including the posterior cingulate cortex (PCC) and anterior cingulate cortex (ACC; Figure 6). For these regions, increases in fMRI response strength were associated with a decreased probability of successful inhibition; or conversely, increases in fMRI strength were linked with an increased probability of error (i.e., failed inhibition).
A

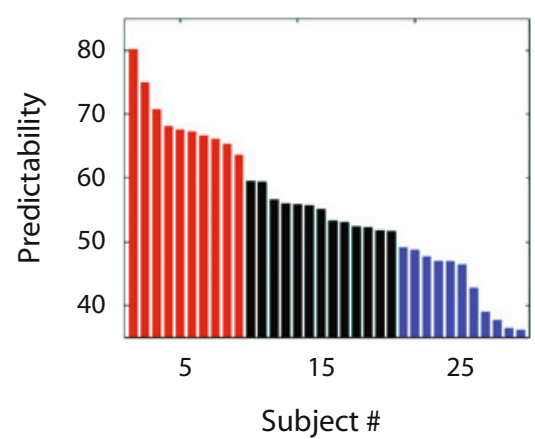

B

R. Caudate

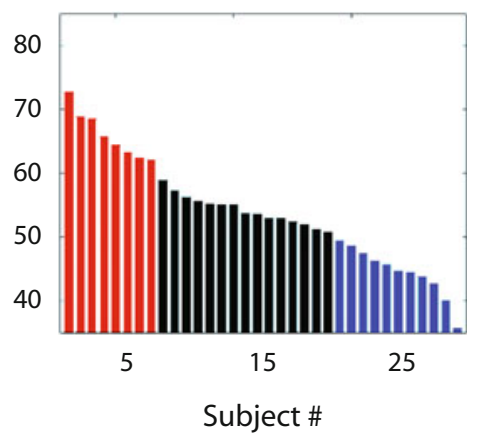

C

R. Putamen

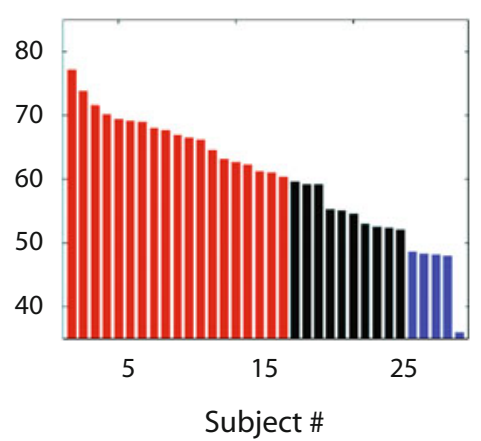

Figure 7. Predictive probability based on fMRI signals. The bar plots illustrate changes in probability for all the subjects in the right inferior frontal gyrus (R. IFG; A), caudate (B), and putamen (C). Note that probabilities were sorted in descending order for plotting purposes. Blue (dark gray) bars indicate the subjects with a predictive probability of less than $50 \%$ (i.e., increases in fMRI response decreased the probability of success), black bars indicate those with values between $50 \%$ and $60 \%$, and red (light gray) bars indicate those with change in predictive probability greater than $60 \%$. 
A

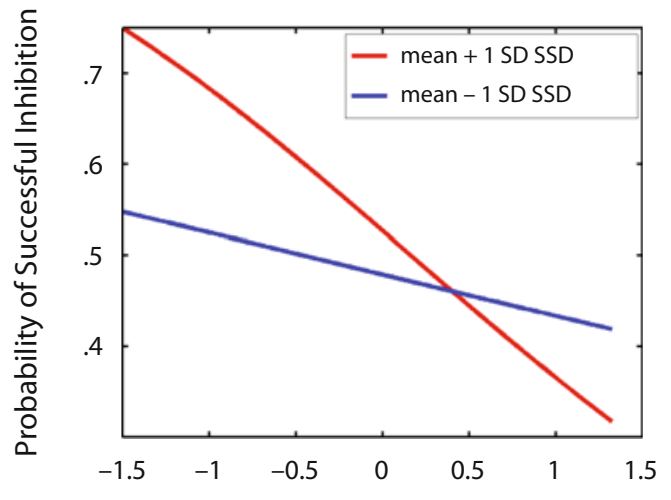

fMRI Amplitude (\%)

\section{B R. Anterior Insula}

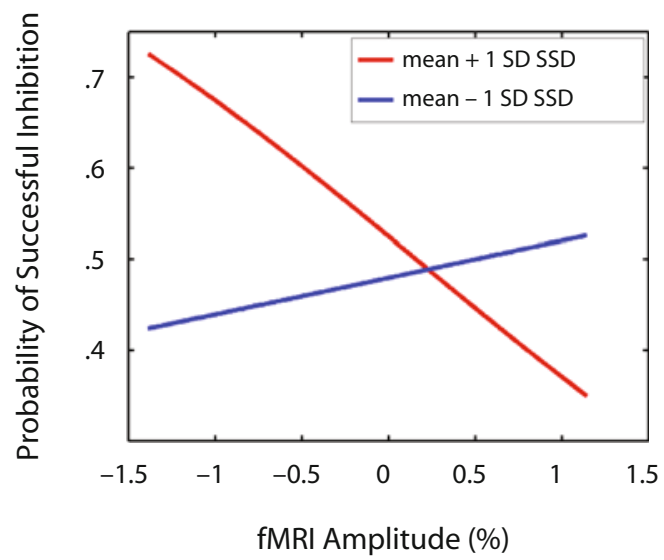

Figure 8. Interaction patterns between fMRI amplitude and stop signal delay (SSD). The probability of successful inhibition was plotted as a function of single-trial fMRI magnitude at two different levels of SSD values, chosen such that they were one standard deviation above and below the mean, respectively. Group-level fits are shown for the anterior cingulate gyrus (A) and right anterior insula (B).

We were interested in further quantifying the strength of the relationship between fMRI magnitude and the probability of successful inhibition. For instance, for the right IFG, when evoked responses changed from medium to high (see the Method section), the probability of successful inhibition increased from $50 \%$ to $55.5 \% \pm 1.89 \%$ $[t(33)=2.88, p<.01]$. Although this figure is relatively modest, plotting the values across individuals (Figure 7A) shows that considerable intersubject variability was present $(S D=11 \%)$. Thus, for instance, predictive probabilities for the 10 top subjects averaged $69.1 \%$. The distribution of predictive probabilities is also shown for the right caudate (Figure 7B) and right putamen (Figure 7C). For the former region, the average value was $53.9 \% \pm 1.49 \%$ $[t(33)=2.62, p<.05 ; S D=8.7 \% ; 64.5 \%$ for the top 10 subjects], and for the latter region, the average value was $60.5 \% \pm 1.55 \%[t(33)=6.75, p<.001 ; S D=9.0 \%$; $70.4 \%$ for the top 10 subjects].

In addition to evaluating the slope corresponding to the magnitude of evoked fMRI responses, we tested the remaining two explanatory variables in the logistic regression model-namely, SSD and the $\mathrm{fMRI} \times \mathrm{SSD}$ interaction. The SSD variable was not statistically significant for any of the regions, whereas the SSD $\times$ fMRI interaction was significant in the ACC and bilateral anterior insula (Table 2). For both of these regions, the link between fMRI responses and behavior depended on the delay, such that at high SSD values, the logistic slope was steeper, as compared with the slope at low SSD values (Figure 8).

The final column of Table 2 displays regions that were reliably engaged during UNSUCC trials (relative to the go baseline). The subthalamic nucleus, a region previously implicated in some response inhibition studies (Aron \& Poldrack, 2006), was engaged during these trials, as well as most brain regions (likely because of the unspecific nature of the contrast between UNSUCC and go trials).

\section{Connectivity Analysis}

What is the relationship between inhibition- and attention-related regions, and how do they vary as a function of behavioral performance? To investigate these questions, we evaluated the correlations between responses in inhibition- and attention-related regions (see the Method section for the definition of these regions). Increased correlations for successful versus unsuccessful performance were observed between the right IFG and bilateral IPS [right IFG and left IPS, $t(33)=2.88, p<.01$; right IFG and right IPS, $t(33)=3.39, p<.005$ ] and between the right IFG and bilateral FEF [right IFG and left FEF, $t(33)=2.54, p<.05$; right $\mathrm{IFG}$ and right FEF, $t(33)=$ $2.55, p<.05]$, as illustrated at the group (Figure 9A) and individual (Figures 9B and 9C) levels; significant differential correlations were not observed between other pairs of regions (e.g., the IPS and caudate).

We further investigated the relationship between interregional evoked responses and behavioral performance in the following way. We reasoned that if fluctuations in responses in frontoparietal regions are important for determining behavior, the strength of the predictive effect between inhibition-related regions and behavior should depend on the strength of these very frontoparietal signals. In particular, we were interested in assessing how the slope of the logistic regression slope between the right IFG and behavior varied as a function of frontoparietal responses (Figure 10A). To do so, trials across subjects were pooled together (to increase statistical power) and binned according to response magnitude independently of behavior (see the Method section). The same trials were then used, and trial-by-trial logistic regressions involving the right IFG and behavior were determined for every bin, as illustrated in Figure 10B. Figure 10C displays the results, which revealed that as response strength in the left IPS increased, the slope of the logistic regression was steeper $[r(22)=.44, p<.05]$ - that is, the link 
A

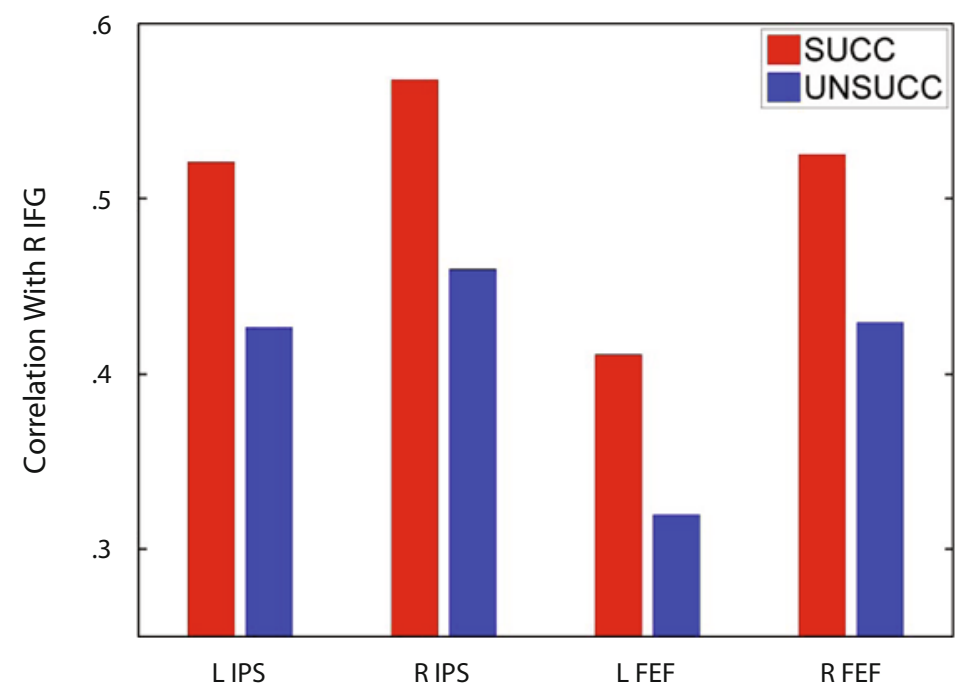

B
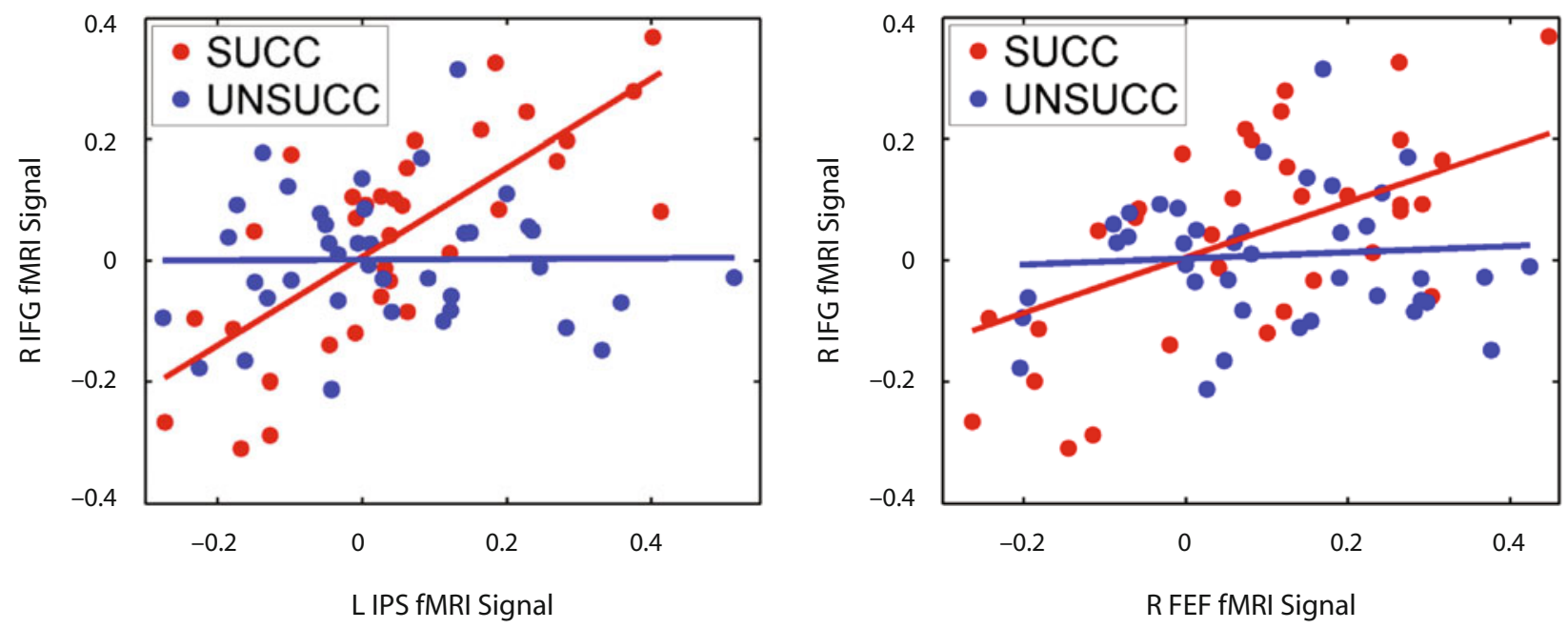

Figure 9. Correlations between right inferior frontal gyrus (IFG) and other frontoparietal regions. (A) At the group level, correlations between the right IFG and intraparietal sulcus (IPS) and between the right IFG and frontal eye field (FEF) increased during successful versus unsuccessful inhibition. $(B, C)$ These correlations are further illustrated for the left IPS and right FEF, respectively, for representative individuals. SUCC, successful stop trial; UNSUCC, unsuccessful stop trial.

between brain responses in the right IFG and behavior was tighter. This type of relationship of the right IFG to behavior was not observed when other frontoparietal regions were considered (i.e., the right IPS, bilateral IPL, and bilateral FEF; $r s<.3$ ). Furthermore, when the caudate or putamen were evaluated (in the place of the right IFG in the analysis shown in Figure 10A), no significant results were detected $(r \mathrm{~s}<.1)$. Finally, when the right IFG and the left IPS were swapped around in the analysis shown in Figure 10A, no significant relationship was observed $[r(22)=.11, p=.62]$, suggesting that the role of these two regions was not completely interchangeable in our task.

\section{DISCUSSION}

In this study, we investigated the link between singletrial fMRI responses and behavioral performance during response inhibition. To do so, subjects performed a stop signal task with stop trials sufficiently spaced apart so as to allow the estimation of single-trial responses. The quantitative assessment of brain-behavior relationships revealed that several cortical and subcortical regions previously implicated in response inhibition parametrically predicted stopping performance on a moment-to-moment basis. Methodologically, our study revealed that the relationship between trial-by-trial responses and behavior 
A

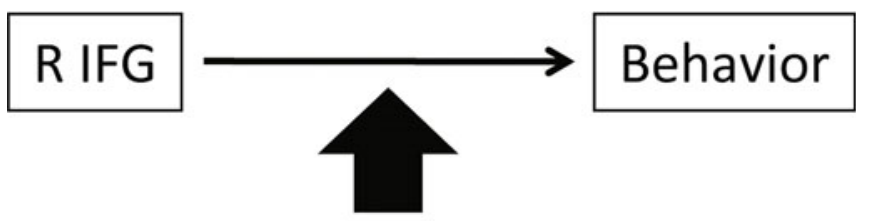

Frontoparietal

(e.g., IPS)

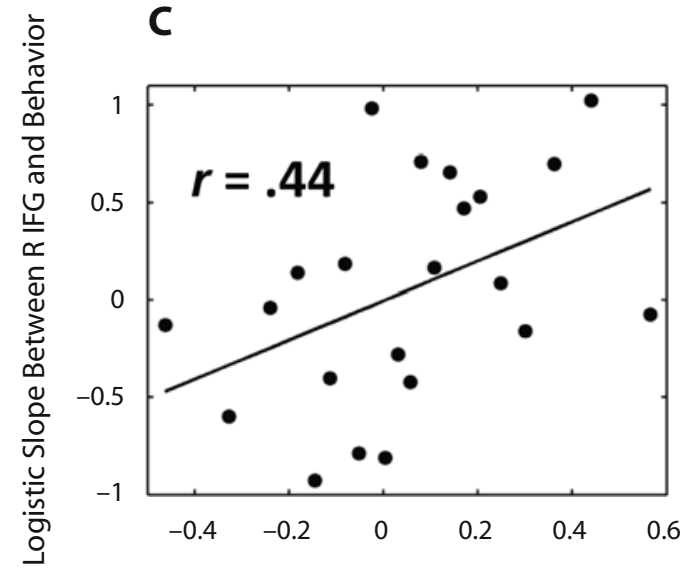

Signal Change: L IPS

B

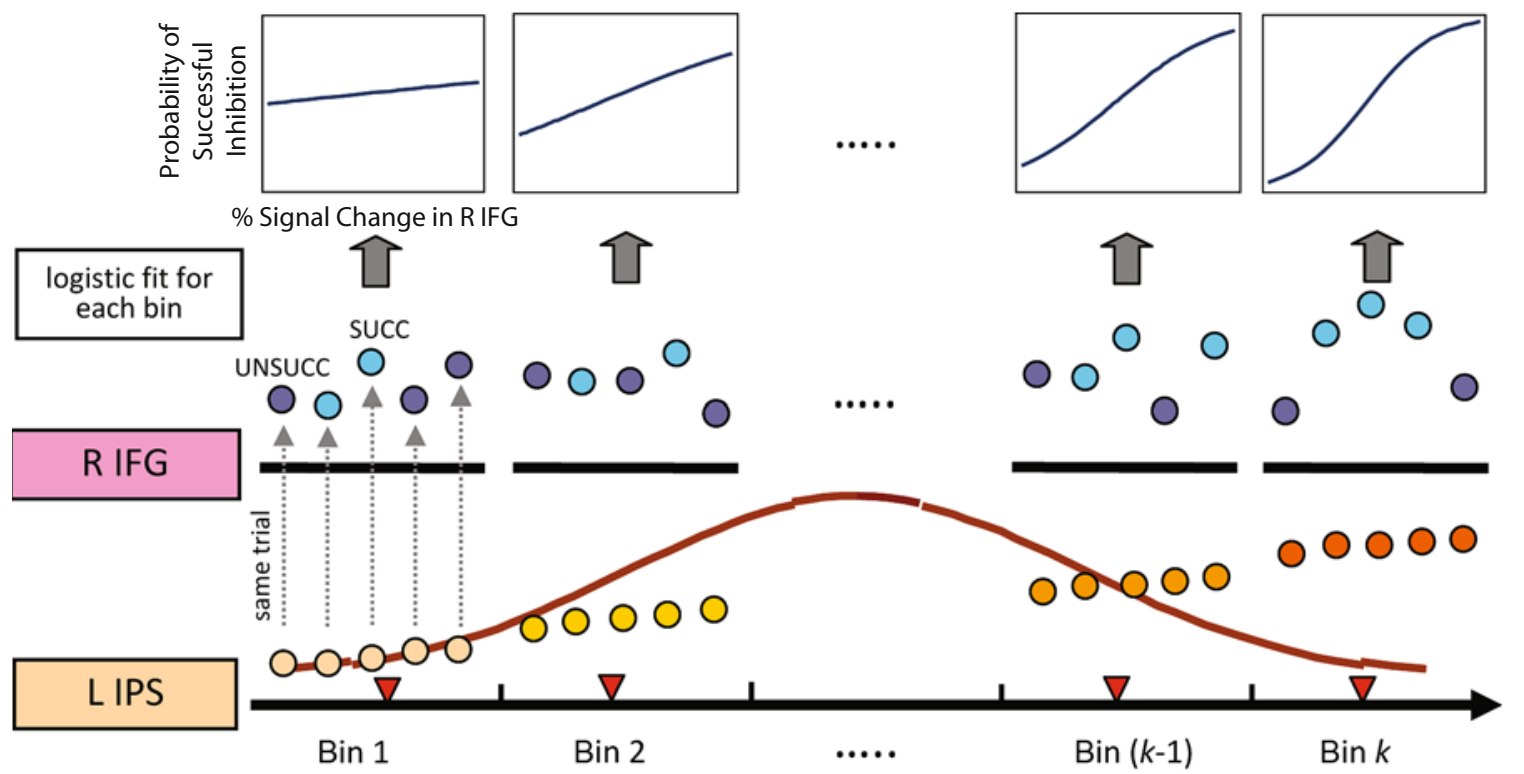

Figure 10. Trial-by-trial interactions between right inferior frontal gyrus (IFG) and other frontoparietal regions and their relationship to behavior. (A) We investigated whether the relationship between the right IFG and behavior varied as a function of the strength of activations in frontoparietal regions (e.g., the intraparietal sulcus [IPS]). (B) Trial-by-trial fluctuations in the left IPS and the magnitude of the brain-behavior relationship in the right IFG. The schematic diagram highlights the main steps in assessing this relationship. The filled circles represent the magnitude of left-IPS (bottom row) and right-IFG (middle row) responses, as indicated via the height from the horizontal bars. Response strength in the left IPS was approximately Gaussian, as indicated via the bell-shaped curve. The top row illustrates idealized logistic regression fits showing an increase in the slope value as a function of the magnitude of evoked responses in the left IPS. The red (gray) triangles indicate the positions of the median response for each left-IPS response bin, which were correlated with the slope of the logistic fit for that same bin. (C) The strength of the right-IFG-to-behavior relationship (as indexed via the slope of the logistic fit) was correlated with the magnitude of evoked responses in the left IPS, such that the stronger the response in left IPS, the tighter the relationship between the right IFG and behavior. SUCC, successful stop trial; UNSUCC, unsuccessful stop trial.

could be detected under experimental conditions similar to those employed to investigate differences in mean responses. In particular, the subjects were scanned during only one session, unlike some other experiments in which multisession data have been utilized (Lim, Padmala, \& Pessoa, 2009; Padmala \& Pessoa, 2008; Ress et al., 2000; Sylvester, Shulman, Jack, \& Corbetta, 2007). Our results thus suggest that cognitive tasks are amenable to trialby-trial analyses with fMRI (Leber et al., 2008; Pessoa et al., 2002).
The stop signal task is a popular behavioral paradigm used to study response inhibition (Verbruggen \& Logan, 2008). It allows for the estimation of the latency of the stop process - namely, the inhibitory $R T$ (i.e., SSRT), which is, by definition, unobserved. In the present study, because we were interested in the variability in trial-bytrial responses, we did not employ a standard fast eventrelated design but, instead, spaced stop trials sufficiently apart that the hemodynamic responses to individual stop trials did not overlap each other. Although this strategy 
did not allow us to estimate responses to go trials (which constituted the baseline condition), the task structure may be better suited to study response inhibition than other fast event-related paradigms that include interspersed null events lasting, say, 2-6 sec, which may induce a more controlled mode of responding. In addition, the strategy of treating go trials as a baseline has been successfully used in the past in both go/no-go (Chikazoe et al., 2009; Garavan et al., 1999; Hester et al., 2009) and stop signal (Chamberlain et al., 2009; Rubia et al., 2003; Rubia et al., 2007) studies.

Previous neuroimaging investigations have adopted two alternative strategies for indexing response inhibitionspecifically, either by contrasting SUCC versus go trials (Aron \& Poldrack, 2006; Xue, Aron, \& Poldrack, 2008) or by contrasting SUCC versus UNSUCC trials (Li et al., 2006; Rubia et al., 2003). The approach of the present study was to probe response inhibition on a trial-by-trial basis by employing logistic regression analysis in terms of a dichotomous behavioral variable (successful, unsuccessful), an approach qualitatively similar to the latter strategy above. The rationale for contrasting SUCC versus go trials is, at times, that the outcome on a stop trial depends mainly on the speed of the go process (Aron \& Poldrack, 2006; Leung \& Cai, 2007). Another reason for contrasting SUCC versus go trials is that SUCC stop trials engage both go and stop processes, such that subtracting the go component would provide an indication of inhibition-related activity (Aron \& Poldrack, 2006; Leung \& Cai, 2007). In this case, however, the inhibition-related response is relatively unspecific, since it includes contributions from various other cognitive processes recruited during the stop signal task, including oddball effects (because of infrequent stop trials), decision making, response selection, and conflict monitoring (Rubia et al., 2003).

The right IFG is believed to be an important brain region for response inhibition, as evidenced by lesion, fMRI, and TMS studies (Aron et al., 2003; Chambers et al., 2007; Chambers et al., 2006; Forstmann et al., 2008; Rubia et al., 2003). Our quantitative brain-behavior analysis is consistent with this notion. Although the magnitude of the relationship was modest at the group level, it was sizable for several subjects. Specifically, at the group level, the probability of successful inhibition increased to $55.5 \%$ for trials with high activation (i.e., top $10 \%$ ), relative to those trials for which subjects were at $50 \%$ performance. At the individual level, for the 10 subjects with the strongest brain-behavior relationships, the same probability increased to $69.1 \%$, on average. It is noteworthy that both group and individual values are comparable to those obtained with neuronal data during perceptual decision tasks with monkeys (Britten, Newsome, Shadlen, Celebrini, \& Movshon, 1996; Purushothaman \& Bradley, 2005), as well as other fMRI studies of decision making (Pessoa \& Padmala, 2005). Somewhat surprisingly, the value of the slope of the fMRI signal in the right IFG was among the lowest observed. In the literature, there is still some dispute as to whether differences between SUCC and UNSUCC trials are present in the right IFG (Aron \& Poldrack, 2006; Rubia et al., 2003). The rela- tively shallow, yet significant, logistic fit observed in our study characterizes how moment-to-moment changes in fMRI responses are linked to changes in behavior and is consistent with the results of previous studies that have reported differences between SUCC and UNSUCC trials in the right IFG based on mean responses (Rubia et al., 2003). Finally, it is important to consider that the slope of the logistic fit reflects the collective contributions of evoked responses to successful inhibitory performance. And because successful behavior in the task relies on several types of processes, including those more closely linked to attention (see below), all of those contributions will influence the steepness of the slope.

Significant positive slopes were also observed in the left precentral gyrus, bilateral caudate, and bilateral putamen. The left precentral gyrus has been implicated in response inhibition (Li et al., 2006) and is also involved in stimulus-response associations (Brass, Wenke, Spengler, \& Waszak, 2009). Converging evidence also indicates the importance of the striatum in response inhibition. For instance, in a lesion study using the stop signal task, lesions of the rat striatum increased the SSRT by $60 \%$ (Eagle \& Robbins, 2003), and a recent stop signal task study reported the involvement of the caudate during inhibition (Li, Yan, Sinha, \& Lee, 2008). Interestingly, hypoactivation in this structure has been documented in clinical populations with inhibitory deficits (Booth et al., 2005). Our results revealed that moment-to-moment fluctuations in response amplitude in these structures were quantitatively linked to the probability of successful inhibition during the stop signal task.

We did not observe evidence for an association between fMRI responses and behavioral performance in the subthalamic nucleus, a subcortical region that has recently been implicated in response inhibition in humans (Aron \& Poldrack, 2006; Ray et al., 2009). In our subjects, this region was equally engaged during SUCC and UNSUCC trials. A possible reason for this negative result is related to the finding that inhibition-related regions may be engaged robustly during UNSUCC trials, too (see Table 2, last column) - but too late to countermand the go response (Garavan, Ross, Murphy, Roche, \& Stein, 2002). If this is the case, it may be difficult to disentangle SUCC versus UNSUCC trials on the basis of fMRI signal amplitude (because of the sluggish nature of the BOLD signal). In a recent lesion study with rats, subthalamic nucleus lesions did not affect the SSRT (Eagle et al., 2008). Instead, the lesions affected stopping accuracy irrespective of the $\mathrm{SSD}$, consistent with the notion that this region may be more strongly involved in the response selection component of the task, and not inhibition per se (Eagle et al., 2008). A similar suggestion about the role of the subthalamic nucleus in response selection was made on the basis of Parkinson's disease patients (van den Wildenberg et al., 2006). The considerations above need to be qualified by the fact that the subthalamic nucleus is a very small structure and that the site observed in the present study might actually not correspond to this nucleus. It is thus possible that the actual subthalamic nucleus was not robustly engaged by our task. 
In the left frontal cortex, we observed significant positive logistic slopes in the IFG and superior frontal gyrus. Although the right IFC is more consistently reported to be involved in response inhibition, the IFC on the left hemisphere has been reported to exhibit differential SUCC versus UNSUCC responses ( $\mathrm{Li}$ et al., 2006; Rubia et al., 2007 ) and has been linked to response inhibition in a recent lesion study (Swick, Ashley, \& Turken, 2008). The left superior frontal gyrus has also been shown to be involved during response inhibition (Li et al., 2006), and patients with left superior frontal cortex lesions exhibit poor performance during go/no-go tasks (Picton et al., 2007). It should be noted that although positive logistic slopes were observed for these regions, unexpectedly, a different pattern of mean responses was observed (relative to, say, those in the right IFG). Specifically, relative to the go baseline, stop-related responses were negative. Because responses to go trials could not be separately estimated in the present study (since they constituted the baseline), the interpretation of these decreased activations is unclear. For instance, it is possible that these regions were engaged by both go and stop processes, but additional studies will be needed to answer this question.

Some regions exhibited significant logistic fits but with negative slopes, including the ACC and PCC. In our design, a negative logistic slope indicated that trials with larger evoked responses were more likely to correspond to failed inhibitions (i.e., errors). The results for the ACC are thus of particular interest, given the role of this region in predicting the likelihood of making an error (Brown \& Braver, 2005), in particular, and error processing more generally (Dehaene, Posner, \& Tucker, 1994; Gehring, Goss, Coles, Meyer, \& Donchin, 1993). The ACC results are also consistent with those obtained in previous response inhibition studies (Garavan, Ross, Kaufman, \& Stein, 2003; Hester, Fassbender, \& Garavan, 2004; Hester et al., 2009; Rubia et al., 2003). The results for the PCC are also consistent with the literature reporting errorrelated responses during response inhibition (Menon, Adleman, White, Glover, \& Reiss, 2001) and/or could be linked to motivational aspects associated with making an error (Maddock, 1999).

We also observed a significant SSD $\times$ fMRI interaction in the ACC. On average, the slope corresponding to the fMRI amplitude variable was negative in this region (higher activity predicted unsuccessful inhibition), and the relationship between fMRI amplitude and probability of inhibition changed on the basis of SSD, such that it was stronger for higher SSD values than for lower ones (see Figure 8A). It is thus possible that activity in the ACC was related to the likelihood of making an error (Brown \& Braver, 2005), because at longer SSD values, subjects would have had a greater chance of committing an error (since the stop command was encountered "too late" for behavioral correction). It is conceivable, however, that the signals observed in the ACC were related to error detection/correction processes (Dehaene et al., 1994; Gehring et al., 1993). However, we believe that this is a less likely possibility, because errors committed at low-SSD trials would have been more salient (because they are more easily correctable) and would be expected to generate stronger error-related responses in the ACC, just the opposite of the pattern observed in our data.

Greater bilateral anterior insula activation during unsuccessful than during successful stop trials has been reported in several response inhibition studies (Hester et al., 2004; Li et al., 2006; Li, Yan, Chao, et al., 2008; Ramautar, Slagter, Kok, \& Ridderinkhof, 2006), but it is unclear whether this activity reflects anticipation and/ or prediction of errors (Preuschoff, Quartz, \& Bossaerts, 2008), or whether it is a consequence of committing errors and is linked to arousal/affective responses subsequent to the errors (Magno, Foxe, Molholm, Robertson, \& Garavan, 2006). Following the same logic as that for the ACC above, our data suggest that the activity in the anterior insula, bilaterally, was more strongly related to predicting or anticipating errors. However, as in the case of the ACC, we cannot rule out posterror effects, and future studies will be needed to clarify the role of the anterior insula during failed inhibitions. No significant interaction effect was detected in the PCC, suggesting that the responses were related to preparing for and making the erroneous response and, hence, did not interact with SSD.

A previous study of the stop signal task also investigated how inhibition-related signals depend on the SSD (Aron \& Poldrack, 2006) and revealed that activations in the subthalamic nucleus and presupplementary motor area were larger for trials with longer SSDs; responses in the IFC did not depend on the SSD. In our study, we did not observe significant $\mathrm{fMRI} \times \mathrm{SSD}$ interactions in these regions. However, the comparison between the two studies is not direct, because Aron and Poldrack employed a different contrast (SUCC vs. go) to index response inhibition.

Although several studies have reported the involvement of parietal regions during response inhibition (Garavan et al., 1999; Hester et al., 2009; Liddle et al., 2001), the exact nature of their involvement remains to be determined. One possibility is that their role is attentional and, in particular, that they reflect trial-by-trial fluctuations in the allocation of the resources (Leber et al., 2008) that are needed for successful behavioral performance during demanding tasks. Consistent with this notion, a recent MEG study revealed that fluctuations of sensory processing linked to both go and stop stimuli impact inhibitory performance during a stop signal task (Boehler et al., 2009). Here, we further investigated the role of attentionrelated processes during response inhibition by focusing on frontoparietal regions that are known to have important roles in attention and that were robustly engaged by the present task - specifically, the IPS, IPL, and FEF. Our analysis revealed that the coupling between these regions and the right IFG was increased during successful versus unsuccessful performance, consistent with the notion that trial-by-trial fluctuations in attention are important in determining the relationship between the right IFG and response inhibition. Interestingly, not all frontoparietal attentional regions behaved in the same way. Although responses in the right IPL exhibited the steepest logistic regression slope (i.e., largest predictive power in terms of SUCC vs. UNSUCC trials), no differential coupling 
between this region and the right IFG was observed. These findings suggest that interactions between the right IFG and attentional regions are relatively specific during the stop signal task. Finally, coupling between frontoparietal regions and the caudate and putamen did not differ as a function of behavioral performance; specifically, similar correlation values were observed during both SUCC and UNSUCC trials.

Evidence that the interactions between frontoparietal attentional regions and the right IFG subserve behavior during the stop signal task was supported by an additional analysis. We reasoned that if responses in attentional regions moderated the impact of the right IFG on behavior, the higher the responses in attentional regions, the stronger the link between the right IFG and behavior (i.e., the steeper the logistic regression slope). Indeed, this pattern was observed when signals from the left IPS were considered (but not when other attentional regions were considered). Although analysis based on fMRI signals, naturally, cannot be used to imply causality, the observation that no correlation was detected when the positions of the left IPS and right IFG were swapped in the analysis suggests that the roles of the two regions was not completely exchangeable in our task. In a related fashion, in a recent stop signal fMRI study, Duann et al. (2009) reported greater functional connectivity between the IFC and presupplementary motor area during SUCC than during UNSUCC trials. Both of these regions are believed to be important nodes of the response inhibition network (Chambers et al., 2009), and our present connectivity analysis complements these findings by revealing greater functional coupling between inhibition and attentional regions during successful response inhibition.

In summary, by employing a trial-based event-related design, our study allowed us to quantitatively assess how moment-to-moment fluctuations in brain responses were associated with the probability of successful performance during a stop signal task. Our findings revealed a network of brain regions whose response variability was tightly coupled with behavioral performance. Among these regions, we observed the right IFG, a region that has been consistently implicated in response inhibition. Other notable regions included the caudate and putamen, bilaterally. Our findings thus support the notion that these regions are important sites of executive control during response inhibition. Furthermore, we investigated how trial-by-trial fluctuations in responses in attentional regions covaried with fluctuations in inhibition-related regions. Accordingly, the coupling between frontoparietal attentional regions and the right IFG increased during successful versus unsuccessful performance, suggesting that efficacious network interactions are important in determining behavioral outcome during the stop signal task. In particular, the link between responses in the right IFG and behavior were moderated by moment-to-moment fluctuations in evoked responses in the left IPS.

\section{AUTHOR NOTE}

Support for this work was provided in part by the National Institute of Mental Health (Grant R01 MH071589) and the Indiana METACyt Initia- tive of Indiana University, funded in part through a major grant from the Lilly Endowment, Inc. We thank Hugh Garavan and other anonymous reviewers for valuable feedback and Andrew Bauer for assistance with figures. Correspondence concerning this article should be addressed to S. Padmala or L. Pessoa, Department of Psychological and Brain Sciences, Indiana University, 1101 East Tenth St., Bloomington, IN 47405 (e-mail: spadmala@indiana.edu or lpessoa@indiana.edu).

\section{REFERENCES}

Aron, A. R., Durston, S., Eagle, D. W., Logan, G. D., Stinear, C. M., \& Stuphorn, V. (2007). Converging evidence for a frontobasal-ganglia network for inhibitory control of action and cognition. Journal of Neuroscience, 27, 11860-11864.

Aron, A. R., Fletcher, P. C., Bullmore, E. T., Sahakian, B. J., \& RobiIns, T. W. (2003). Stop-signal inhibition disrupted by damage to right inferior frontal gyrus in humans. Nature Neuroscience, 6, 115116.

Aron, A. R., \& Poldrack, R. A. (2006). Cortical and subcortical contributions to stop signal response inhibition: Role of the subthalamic nucleus. Journal of Neuroscience, 26, 2424-2433.

Boehler, C. N., Münte, T. F., Krebs, R. M., Heinze, H.-J., SchoenFELD, M. A., \& Hopf, J.-M. (2009). Sensory MEG responses predict successful and failed inhibition in a stop-signal task. Cerebral Cortex, 19, 134-145.

Booth, J. R., Burman, D. D., Meyer, J. R., Lei, Z., Trommer, B. L., DAVENPORT, N. D., ET AL. (2005). Larger deficits in brain networks for response inhibition than for visual selective attention in attention deficit hyperactivity disorder (ADHD). Journal of Child Psychology \& Psychiatry, 46, 94-111.

Boucher, L., Palmeri, T. J., Logan, G. D., \& Schall, J. D. (2007). Inhibitory control in mind and brain: An interactive race model of countermanding saccades. Psychological Review, 114, 376-397.

Brass, M., Derrfuss, J., Forstmann, B., \& von Cramon, D. Y. (2005). The role of the inferior frontal junction area in cognitive control. Trends in Cognitive Sciences, 9, 314-316.

Brass, M., WenKe, D., SPEngler, S., \& WaszaK, F. (2009). Neural correlates of overcoming interference from instructed and implemented stimulus-response associations. Journal of Neuroscience, 29, 17661772.

Britten, K. H., Newsome, W. T., Shadlen, M. N., Celebrini, S., \& Movshon, J. A. (1996). A relationship between behavioral choice and the visual responses of neurons in macaque MT. Visual Neuroscience, 13, 87-100.

Brown, J. W., \& Braver, T. S. (2005). Learned predictions of error likelihood in the anterior cingulate cortex. Science, 307, 1118-1121.

Casey, B. J., Trainor, R. J., Orendi, J. L., Schubert, A. B., Nystrom, L. E., GIEDD, J. N., ET AL. (1997). A developmental functional MRI study of prefrontal activation during performance of a go-no-go task. Journal of Cognitive Neuroscience, 9, 835-847.

Chamberlain, S. R., Hampshire, A., Müller, U., Rubia, K., Del CAMPo, N., Craig, K., ET AL. (2009). Atomoxetine modulates right inferior frontal activation during inhibitory control: A pharmacological functional magnetic resonance imaging study. Biological Psychiatry, 65, 550-555.

Chambers, C. D., Bellgrove, M. A., Gould, I. C., English, T., GaRaVAn, H., McNaught, E., ET AL. (2007). Dissociable mechanisms of cognitive control in prefrontal and premotor cortex. Journal of Neurophysiology, 98, 3638-3647.

Chambers, C. D., Bellgrove, M. A., Stokes, M. G., Henderson, T. R., Garavan, H., Robertson, I. H., et al. (2006). Executive "brake failure" following deactivation of human frontal lobe. Journal of Cognitive Neuroscience, 18, 444-455.

Chambers, C. D., Garavan, H., \& Bellgrove, M. A. (2009). Insights into the neural basis of response inhibition from cognitive and clinical neuroscience. Neuroscience \& Biobehavioral Reviews, 33, 631-646.

Chen, C.-Y., Muggleton, N. G., Tzeng, O. J. L., Hung, D. L., \& JuAn, C.-H. (2009). Control of prepotent responses by the superior medial frontal cortex. NeuroImage, 44, 537-545.

Chikazoe, J., Jimura, K., Asari, T., Yamashita, K., Morimoto, H., Hirose, S., ET AL. (2009). Functional dissociation in right inferior frontal cortex during performance of go/no-go task. Cerebral Cortex, 19, 146-152. 
Cohen, M. S. (1997). Parametric analysis of fMRI data using linear systems methods. NeuroImage, 6, 93-103.

Colzato, L. S., van den Wildenberg, W. P., \& Hommel, B. (2007). Impaired inhibitory control in recreational cocaine users. PLOS ONE, 2, e1143.

Corbetta, M., \& Shulman, G. L. (2002). Control of goal-directed and stimulus-driven attention in the brain. Nature Reviews Neuroscience, 3, 201-215.

Cox, R. W. (1996). AFNI: Software for the analysis and visualization of functional magnetic resonance neuroimages. Computers \& Biomedical Research, 29, 162-173.

Dehaene, S., Posner, M. I., \& Tucker, D. M. (1994). Localization of a neural system for error detection and compensation. Psychological Science, , 5, 303-305.

Duann, J.-R., IDE, J. S., Luo, X., \& Li, C.-S. R. (2009). Functional connectivity delineates distinct roles of the inferior frontal cortex and presupplementary motor area in stop signal inhibition. Journal of Neuroscience, 29, 10171-10179.

Eagle, D. M., Baunez, C., Hutcheson, D. M., Lehmann, O., Shah, A. P., \& Robiins, T. W. (2008). Stop-signal reaction-time task performance: Role of prefrontal cortex and subthalamic nucleus. Cerebral Cortex, 18, 178-188.

EAgLe, D. M., \& Robbins, T. W. (2003). Inhibitory control in rats performing a stop-signal reaction-time task: Effects of lesions of the medial striatum and $d$-amphetamine. Behavioral Neuroscience, 117, $1302-1317$

EIMER, M. (1993). Effects of attention and stimulus probability on ERPs in a Go/Nogo task. Biological Psychology, 35, 123-138.

Floden, D., \& Stuss, D. T. (2006). Inhibitory control is slowed in patients with right superior medial frontal damage. Journal of Cognitive Neuroscience, 18, 1843-1849.

Forstmann, B. U., Jahfari, S., Scholte, H. S., Wolfensteller, U., VAN DEN Wildenberg, W. P. M., \& RidDerinkHof, K. R. (2008). Function and structure of the right inferior frontal cortex predict individual differences in response inhibition: A model-based approach. Journal of Neuroscience, 28, 9790-9796.

Garavan, H., Ross, T. J., Kaufman, J., \& Stein, E. A. (2003). A midline dissociation between error-processing and response-conflict monitoring. Neurolmage, 20, 1132-1139.

Garavan, H., Ross, T. J., Murphy, K., Roche, R. A. P., \& Stein, E. A. (2002). Dissociable executive functions in the dynamic control of behavior: Inhibition, error detection, and correction. NeuroImage, 17, 1820-1829.

Garavan, H., Ross, T. J., \& Stein, E. A. (1999). Right hemispheric dominance of inhibitory control: An event-related functional MRI study. Proceedings of the National Academy of Sciences, 96, 83018306.

Gehring, W., Goss, B., Coles, M., Meyer, D., \& Donchin, E. (1993). A neural system for error detection and compensation. Psychological Science, 4, 385-390.

Gelman, A., \& Hill, J. (2007). Data analysis using regression and multilevel/hierarchical models. Cambridge: Cambridge University Press.

Genovese, C. R., LaZar, N. A., \& Nichols, T. (2002). Thresholding of statistical maps in functional neuroimaging using the false discovery rate. NeuroImage, $\mathbf{1 5}, 870-878$.

Hester, R., Fassbender, C., \& Garavan, H. (2004). Individual differences in error processing: A review and reanalysis of three eventrelated fMRI studies using the GO/NOGO task. Cerebral Cortex, 14, 986-994.

Hester, R., Madeley, J., Murphy, K., \& Mattingley, J. B. (2009). Learning from errors: Error-related neural activity predicts improvements in future inhibitory control performance. Journal of Neuroscience, 29, 7158-7165.

Hosmer, D. W., \& Lemeshow, S. (2000). Applied logistic regression (2nd ed.). New York: Wiley.

Kalaska, J. F., \& Crammond, D. J. (1995). Deciding not to GO: Neuronal correlates of response selection in a $\mathrm{GO} / \mathrm{NOGO}$ task in primate premotor and parietal cortex. Cerebral Cortex, 5, 410-428.

Kastner, S., \& Ungerleider, L. G. (2001). The neural basis of biased competition in human visual cortex. Neuropsychologia, 39, 12631276 .
Kriegeskorte, N., Simmons, W. K., Bellgowan, P. S., \& Baker, C. I. (2009). Circular analysis in systems neuroscience: The dangers of double dipping. Nature Neuroscience, 12, 535-540.

Leber, A. B., Turk-Browne, N. B., \& Chun, M. M. (2008). Neural predictors of moment-to-moment fluctuations in cognitive flexibility. Proceedings of the National Academy of Sciences, 105, 13592-13597.

LEUNG, H.-C., \& CAI, W. (2007). Common and differential ventrolateral prefrontal activity during inhibition of hand and eye movements. Journal of Neuroscience, 27, 9893-9900.

Li, C.-S. R., Huang, C., Constable, R. T., \& Sinha, R. (2006). Imaging response inhibition in a stop-signal task: Neural correlates independent of signal monitoring and post-response processing. Journal of Neuroscience, 26, 186-192.

Li, C.-S. R., Yan, P., Chao, H. H., Sinha, R., Paliwal, P., Constable, R. T., ET AL. (2008). Error-specific medial cortical and subcortical activity during the stop signal task: A functional magnetic resonance imaging study. Neuroscience, 155, 1142-1151.

Li, C.-S. R., Yan, P., Sinha, R., \& LeE, T.-W. (2008). Subcortical processes of motor response inhibition during a stop signal task. NeuroImage, 41, 1352-1363.

LidDle, P. F., KIEHL, K. A., \& SMITH, A. M. (2001). Event-related fMRI study of response inhibition. Human Brain Mapping, 12, 100-109.

Lim, S. L., Padmala, S., \& Pessoa, L. (2009). Segregating the significant from the mundane on a moment-to-moment basis via direct and indirect amygdala contributions. Proceedings of the National Academy of Sciences, 106, 16841-16846.

LofTus, G. R., \& Masson, M. E. (1994). Using confidence intervals in within-subject designs. Psychonomic Bulletin \& Review, 1, 476-490.

LogAN, G. D. (1994). On the ability to inhibit thought and action: A user's guide to the stop signal paradigm. In D. Dagenbach \& T. H. Carr (Eds.), Inhibitory processes in attention, memory, and language (pp. 189-239). San Diego: Academic Press.

Logan, G. D., \& CowAn, W. B. (1984). On the ability to inhibit thought and action: A theory of an act of control. Psychological Review, 91, 295-327.

Logan, G. D., SchaChaR, R. J., \& TANNOCK, R. (1997). Impulsivity and inhibitory control. Psychological Science, 8, 60-64.

MADDOCK, R. J. (1999). The retrosplenial cortex and emotion: New insights from functional neuroimaging of the human brain. Trends in Neurosciences, 22, 310-316.

Magno, E., Foxe, J. J., Molholm, S., Robertson, I. H., \& GaravAN, H. (2006). The anterior cingulate and error avoidance. Journal of Neuroscience, 26, 4769-4773.

Menon, V., Adleman, N. E., White, C. D., Glover, G. H., \& Reiss, A. L. (2001). Error-related brain activation during a go/nogo response inhibition task. Human Brain Mapping, 12, 131-143.

Nachev, P., Wydell, H., O'Neill, K., Husain, M., \& Kennard, C. (2007). The role of the pre-supplementary motor area in the control of action. NeuroImage, 36(Suppl. 2), T155-T163.

Padmala, S., \& Pessoa, L. (2008). Affective learning enhances visual detection and responses in primary visual cortex. Journal of Neuroscience, 28, 6202-6210.

Padmala, S., \& Pessoa, L. (2010). Interactions between cognition and motivation during response inhibition. Neuropsychologia, 48, 558565.

Pessoa, L., Gutierrez, E., Bandettini, P. B., \& Ungerleider, L. G. (2002). Neural correlates of visual working memory: fMRI amplitude predicts task performance. Neuron, 35, 975-987.

PeSSOA, L., \& PADMALA, S. (2005). Quantitative prediction of perceptual decisions during near-threshold fear detection. Proceedings of the $\mathrm{Na}$ tional Academy of Sciences, 102, 5612-5617.

Pessoa, L., \& Ungerleider, L. G. (2004). Top-down mechanisms for working memory and attentional processes. In M. S. Gazzaniga (Ed.), The new cognitive neurosciences (3rd ed., pp. 919-930). Cambridge, MA: MIT Press.

Picton, T. W., Stuss, D. T., Alexander, M. P., Shallice, T., Binns, M. A., \& Gillingham, S. (2007). Effects of focal frontal lesions on response inhibition. Cerebral Cortex, 17, 826-838.

Preuschoff, K., Quartz, S. R., \& Bossaerts, P. (2008). Human insula activation reflects risk prediction errors as well as risk. Journal of Neuroscience, 28, 2745-2752.

Purushothaman, G., \& Bradley, D. C. (2005). Neural population 
code for fine perceptual decisions in area MT. Nature Neuroscience, $\mathbf{8}, 99-106$.

Ramautar, J. R., Slagter, H. A., Kok, A., \& Ridderinkhof, K. R. (2006). Probability effects in the stop-signal paradigm: The insula and the significance of failed inhibition. Brain Research, 1105, 143-154.

RATCLIFFE, S. J., \& ShUlTs, J. (2008). GEEQBOX: A MATLAB toolbox for generalized estimating equations and quasi-least squares. Journal of Statistical Software, 25, 1-14.

Ray, N. J., Jenkinson, N., Brittain, J., Holland, P., Joint, C., NANDI, D., ET AL. (2009). The role of the subthalamic nucleus in response inhibition: Evidence from deep brain stimulation for Parkinson's disease. Neuropsychologia, 47, 2828-2834.

Ress, D., Backus, B. T., \& Heeger, D. J. (2000). Activity in primary visual cortex predicts performance in a visual detection task. Nature Neuroscience, 3, 940-945.

Rubia, K., Smith, A. B., Brammer, M. J., \& Taylor, E. (2003). Right inferior prefrontal cortex mediates response inhibition while mesial prefrontal cortex is responsible for error detection. NeuroImage, 20, 351-358.

Rubia, K., Smith, A. B., Taylor, E., \& Brammer, M. (2007). Linear age-correlated functional development of right inferior frontostriato-cerebellar networks during response inhibition and anterior cingulate during error-related processes. Human Brain Mapping, 28, 1163-1177.

Swick, D., Ashley, V., \& Turken, A. U. (2008). Left inferior frontal gyrus is critical for response inhibition. BMC Neuroscience, 9, 102.

Sylvester, C. M., Shulman, G. L., Jack, A. I., \& Corbetta, M. (2007). Asymmetry of anticipatory activity in visual cortex predicts the locus of attention and perception. Journal of Neuroscience, 27, 14424-14433.

TAlairach, J., \& Tournoux, P. (1988). A co-planar stereotaxic atlas of the human brain. New York: Thieme.
VAn den Wildenberg, W. P., van Boxtel, G. J., van der Molen, M. W., Bosch, D. A., Speelman, J. D., \& Brunia, C. H. (2006). Stimulation of the subthalamic region facilitates the selection and inhibition of motor responses in Parkinson's disease. Journal of Cognitive Neuroscience, 18, 626-636.

Verbruggen, F., \& Logan, G. D. (2008). Response inhibition in the stop-signal paradigm. Trends in Cognitive Sciences, 12, 418-424.

Vink, M., Kahn, R. S., Raemaekers, M., van den Heuvel, M., Boersma, M., \& Ramsey, N. F. (2005). Function of striatum beyond inhibition and execution of motor responses. Human Brain Mapping, 25, 336-344.

Williams, B., Ponesse, J., Schachar, R., Logan, G., \& Tannock, R. (1999). Development of inhibitory control across the life span. Developmental Psychology, 35, 205-213.

Xue, G., Aron, A. R., \& Poldrack, R. A. (2008). Common neural substrates for inhibition of spoken and manual responses. Cerebral Cortex, 18, 1923-1932.

Zeger, S. L., \& Liang, K. Y. (1986). Longitudinal data analysis for discrete and continuous outcomes. Biometrics, 42, 121-130.

Zhou, D., Thompson, W. K., \& Siegle, G. (2009). MATLAB toolbox for functional connectivity. NeuroImage, 47, 1590-1607.

\section{SUPPLEMENTAL MATERIALS}

A supplemental figure with the results of a simulation study on selection bias may be downloaded from http://cabn.psychonomic-journals .org/content/supplemental.

(Manuscript received August 24, 2009; revision accepted for publication November 17, 2009.) 